\title{
EL ICNOGÉNERO Spongeliomorpha EN EL NEÓGENO SUPERIOR DE LA CUENCA DEL GUADALQUIVIR (ÁREA DE LEPE-AYAMONTE, HUELVA, ESPAÑA)
}

\author{
Fernando MUNIIZ y Eduardo MAYORAL \\ Departamento de Geodinámica y Paleontología, Facultad de \\ Ciencias Experimentales, Universidad de Huelva, 21819, Palos de la \\ Frontera, Huelva.
}

Muñiz, F. y Mayoral, E. 2001. El icnogénero Spongeliomorpha en el Neógeno superior de la Cuenca del Guadalquivir (área de Lepe-Ayamonte, Huelva, España. [The ichnogenus Spongeliomorpha from the upper Neogene of the Guadalquivir Basin (Lepe-Ayamonte area, Huelva, Spain.] Revista Espainola de Paleontología, 16(1), 115-130. ISSN 0213-6937.

\begin{abstract}
The marine Upper Neogene siliciclastic sediments in the southwestern sector of the Guadalquivir Basin (LepeAyamonte area, Huelva, SW of Spain) are characterized by a high ichnological content. The ichnological study has revealed the occurrence of several trace fossils related to the ichnogenus Spongeliomorpha. The ichnospecies recognized are: Spongeliomorpha sicula D'Alessandro and Bromley, 1995, S. chevronensis nov. ichnosp. and S. sinuostriata nov. ichnosp. These ichnospecies are characterized by a strong ornamentation due to deep raker impressions. So, S. chevronensis presents a chevron-like pattern and S. sinuostriata shows a sinuous to sigmoidal network. Finally, another bad preserved trace fossil have been identified as Spongeliomorpha ichnosp. indet.

The ichnospecies described are in relation to the work of decapod crustaceans produced in a marine environment (estuary/bay) characterized by a silty to muddy consolidated bottom with an important content in organic matter. Ethologically are classified as fodinichnia trace fossils or agrichnion in the case of Spongeliomorpha sicula ichnospecies.
\end{abstract}

Keywords: Spongeliomorpha, Spongeliomorpha chevronensis nov. ichnosp., Spongeliomorpha sinuostriata nov. ichnosp., upper Neogene, Guadalquivir Basin, Huelva, Spain.

\section{RESUMEN}

Los depósitos siliciclásticos marinos neógenos del sector suroccidental de la Cuenca del Guadalquivir (área de Lepe-Ayamonte, Huelva, SO de España) se caracterizan por un alto contenido paleoicnológico. Su estudio ha permitido reconocer varias estructuras etológicas asignables al icnogénero Spongeliomorpha. Se ha reconocido Spongeliomorpha sicula D'Alessandro y Bromley, 1995 y se han definido dos icnoespecies nuevas, Spongeliomorpha chevronensis nov. ichnosp. y S. sinuostriata nov. ichnosp., ambas caracterizadas por presentar las paredes externas fuertemente ornamentadas por marcas de rastrillaje dispuestas en modelos diferentes: Spongeliomorpha chevronensis presenta un patrón ornamental en forma de espiga y S. sinuostriata, un trazado sinuoso, donde las marcas se yuxtaponen entre sí, para formar un enrejado sigmoidal. Por último, se ha identificado otra pista, cuya asignación sistemática es incierta, Spongeliomorpha ichnosp. indet., ya que su estado de conservación no permite hacer mayores precisiones.

Las icnoespecies descritas se relacionan con la actividad excavadora de crustáceos decápodos en un medio marino restringido (bahia/estuario), de fondos limo-fangosos, consolidados y con abundante materia orgánica. Desde el punto de vista etológico, se han clasificado como pistas de búsqueda de alimento (fodinicnia) o de construcción de una granja/trampa (agricnion) en el caso particular de $S$. sicula.

Palabras clave: Spongeliomorpha, Spongeliomorpha chevronensis nov. ichnosp., Spongeliomorpha sinuostriata nov. ichnosp., Neógeno superior, Cuenca del Guadalquivir, Huelva, España. 


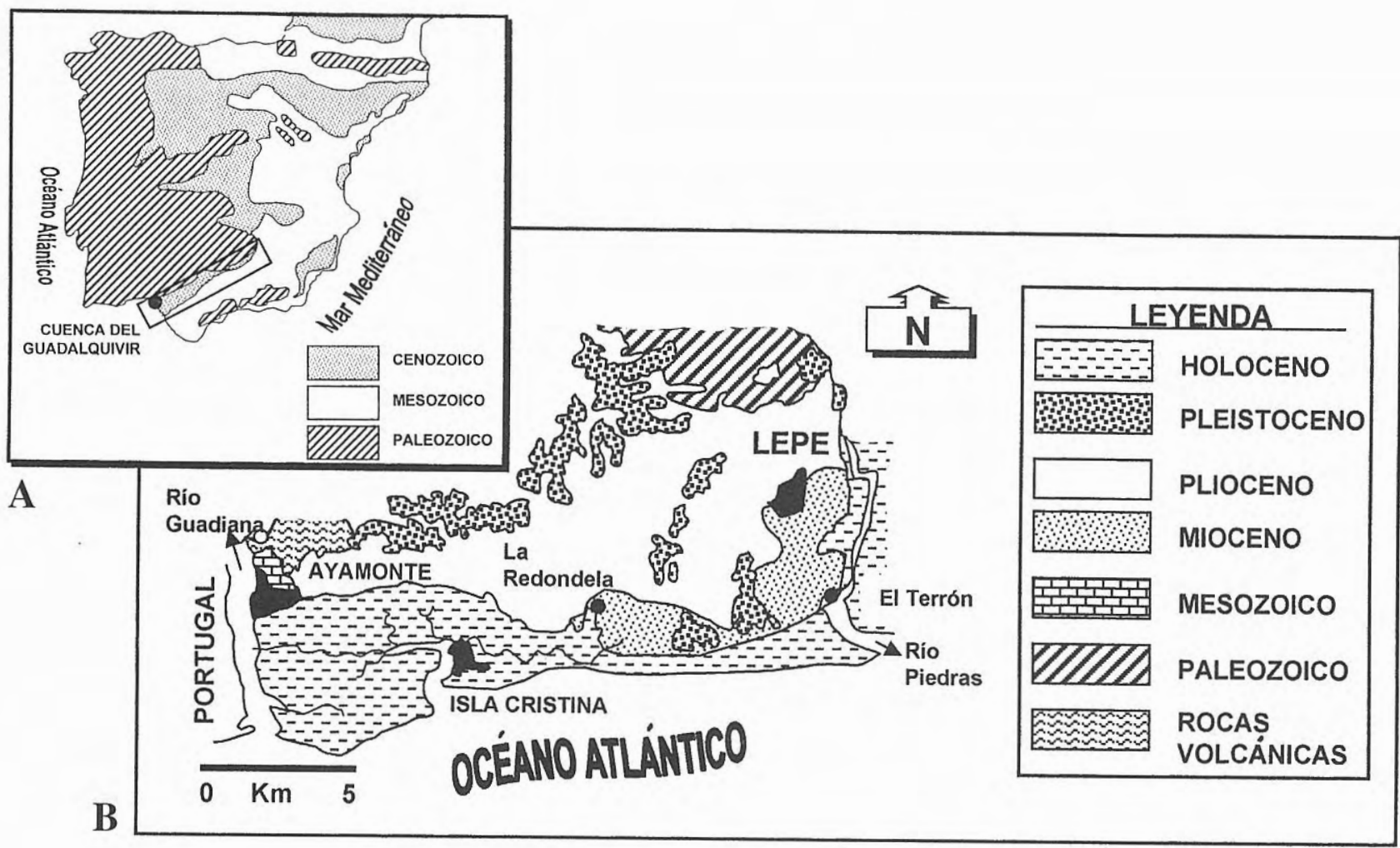

Figura 1. Situación geográfica y contexto geológico del área estudiada. A. Localización dentro de la Cuenca del Guadalquivir. B. Esquema geológico del área Lepe-Ayamonte.

Geographical and geological setting of the studied zone. A. Location in the Guadalquivir Basin. B. Geological map of the Lepe-Ayamonte area.

\section{INTRODUCCIÓN}

El sector suroccidental de la Cuenca del Guadalquivir está representado por unidades siliciclásticas marinas, cuya edad determinada, hasta el momento, abarca desde el Mioceno Superior (Tortoniense superior-Messiniense) al Plioceno Inferior (Zancliense superior). El estudio del contenido paleoicnológico (Muñiz Guinea, 1998) de estas unidades ha permitido diferenciar varias icnoespecies pertenecientes al icnogénero Spongeliomorpha Saporta, 1887: Spongeliomorpha chevronensis nov. ichnosp., S. sinuostriata nov. ichnosp. y S. sicula D'Alessandro y Bromley, 1995, dos de las cuales son nuevas. En el presente trabajo se analiza la taxonomía de estas icnoespecies y se discute los posibles organismos productores, así como las consideraciones paleoambientales que se derivan de su estudio.

\section{MATERIAL ESTUDIADO}

Los icnofósiles estudiados proceden de diversas secciones estratigráficas del sector suroccidental de la Depresión del Guadalquivir, entre los términos municipales de Lepe y Ayamonte (provincia de Huelva) (Fig. 1).

Desde el punto de vista geológico, los materiales estudiados se encuentran en el extremo suroccidental de la Cuenca del Guadalquivir (Fig. 1) y se corresponden con depósitos marinos pertenecientes tanto al Cortejo
Sedimentario Transgresivo que se produce durante el Mioceno Superior como al Cortejo Regresivo que tuvo lugar durante el Plioceno Inferior y Superior (Sierro et al., 1990; Cachão, 1995; Muñiz Guinea, 1998). Estos materiales se depositaron en discordancia erosiva y angular sobre el sustrato paleozoico (Carbonífero inferior), representado principalmente por pizarras y grauwacas en facies Culm. A su vez, suprayacentes a estos materiales neógenos y carboníferos, se apoyan en discordancia erosiva otros relacionados con diferentes niveles de terrazas fluviales de edad cuaternaria (Pleistoceno Inferior) (Cáceres Puro, 1999). La serie estratigráfica global para esta área consta de muro hacia techo de diez conjuntos litológicos o tramos (Fig. 2):

TRAMO 1: Arcillas grises, oscuras a negras, de 2 a $4 \mathrm{~m}$ de espesor visible. Estas arcillas, ligeramente arenosas, son ricas en materia orgánica. No se observan estructuras sedimentarias. El contenido fósil está constituido por bivalvos (nuculánidos, mitílidos, pectínidos, cardíidos, tellínidos, venéridos y corbúlidos) generalmente de pequeño tamaño, gasterópodos (turritélidos, capúlidos, natícidos y cónidos), escafópodos (dentálidos) y equinoideos (Schizaster sp.). También es muy común la presencia del foraminífero bentónico Cyclammina sp., junto con restos carbonosos. Localmente se encuentran niveles intensamente bioturbados, reconociéndose un contenido icnológico formado por ocho icnoespecies. El conjunto presenta hacia techo niveles más limosos de color blanco, que vienen a señalar el paso gradual al siguiente conjunto litológico.

TRAMO 2: limos arenosos, blancos, con una potencia que 


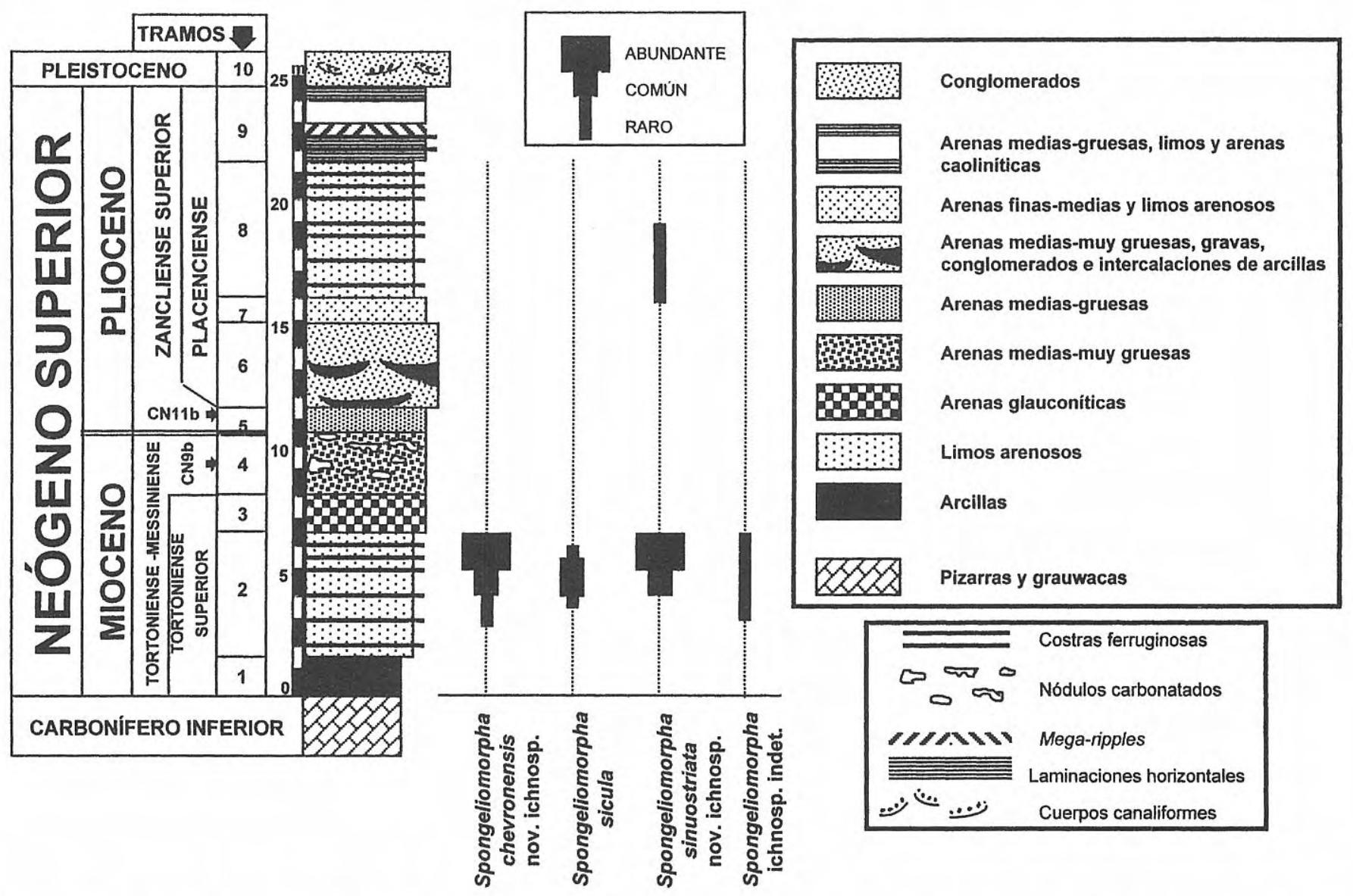

Figura 2. Columna estratigráfica global del área Lepe-Ayamonte con la localización y abundancia relativa de las icnoespecies estudiadas.

Stratigraphic section of the Lepe-Ayamonte area showing the location and relative abundance of studied ichnospecies.

varía entre 5 y $14 \mathrm{~m}$, dispuestos de forma aparentemente concordante sobre el tramo infrayacente. Presentan intercalaciones arcillo-limosas, grises oscuras, y arenas finas, de espesor decimétrico. En estos niveles arenosos son frecuentes las laminaciones paralelas, horizontales y suavemente inclinadas en sentidos opuestos, así como intercalaciones de niveles microconglomeráticos de escala centimétrica en la parte superior del conjunto. La característica distintiva de este tramo es la presencia de numerosas costras ferruginosas relacionadas con superficies de no deposito o diastemas (Mayoral y Muñiz, 1994, 1995 y 1996). Éstas constituyen superficies de carácter lumaquélico, compuestas principalmente por malacofauna: bivalvos (nuculánidos, árcidos, gliciméridos, mitílidos, pínnidos, pectínidos, anomíidos, ostreidos, lucínidos, astártidos, cardíidos, máctridos, solénidos, tellínidos, donácidos, solecúrtidos, venéridos, corbúlidos, hiatélidos), gasterópodos (turritélidos, capúlidos, aporráidos, natícidos, tónnidos, murícidos, olivínidos, cancelaríidos, cónidos), escafópodos (dentálidos), cirrípedos balanomorfos, foraminíferos bentónicos, dientes de seláceos; así como fragmentos de própodos de crustáceos decápodos, un cefalópodo sepioideo [Sepia (Parasepia) melendezi (Mayoral y Muñiz, 1994)] y abundantes restos de cetáceos (vértebras y costillas principalmente). También suelen presentar moldes de crecimiento de sales, septarias y estructuras de escape de gases o fluidos. En ocasiones, las superficies anteriores pasan lateralmente a horizontes intensamente bioturbados y/o bioerosionados. La bioturbación también está presente en los niveles limosos, con un número de icnotaxones muy alto.

TRAMO 3: Arenas glauconíticas, de color verdoso con tintes anaranjados. La potencia del tramo oscila entre 2 y 3 $\mathrm{m}$, hacia el oeste cambia lateralmente de facies a unas arenas medias, de color pardo-anaranjado, con una intensa laminación paralela horizontal. Se disponen en aparente concordancia sobre el tramo anterior. Presentan laminaciones paralelas horizontales y la concentración de glauconita decrece, en general, hacia techo. Se han encontrado restos de fauna, bivalvos y gasterópodos, principalmente, y un contenido icnológico significativo representado por cinco icnoespecies. Por otro lado, la glauconita ha sido datada radiométricamente por el método $\mathrm{K} / \mathrm{Ar}$, resultando una edad de $-6,6 \pm 0,3$ y $-6,7 \pm 0,3 \mathrm{Ma}$ (Galán et al., 1995). Esta datación sitúa a todos los conjuntos litológicos anteriores en una posición cronoestratigráfica de, al menos, Tortoniense superior (Mayoral y Muñiz, 1995).

TRAMO 4: Arenas medias-muy gruesas, pardoamarillentas, de 1 a $4 \mathrm{~m}$ de espesor visible, con nódulos carbonatados en su parte inferior y superior, así como 
niveles cementados por carbonato cálcico, más continuos en su parte media. El contenido fosilífero se encuentra principalmente en los nódulos y niveles carbonatados, destacando la presencia de bivalvos, como Pholadomya alpina (Matheron) y Crassostrea aff. gryphoides (Schlotheim), gasterópodos [entre los que se encuentran Conus (Leptoconus) aff. allioni (Michelotti) y Conus (Dendroconus) berghausi (Sacco)], cirrípedos balanomorfos, abundantes dientes de pisciformes condrictios y restos de cetáceos. El contenido icnológico es muy pobre y está representado únicamente por la icnoespecie Ophiomorpha nodosa (Lundgren). Los niveles más arenosos son ricos en nanoplancton calcáreo, perteneciente a la biozona CN9b (Okada y Bukry, 1980), lo que permite atribuir su edad al Tortoniense inferior-Messiniense (Muñiz Guinea, 1998).

TRAMO 5: Arenas medias-muy gruesas, amarillentas, de $1 \mathrm{~m}$ de espesor visible. Su contenido malacológico fósil es muy rico, representado por bivalvos (similares a los del Tramo 2, junto con nucúlidos, ungúlidos, chámidos, leptónidos, semélidos, solecúrtidos y miidos), gasterópodos (tróchidos, nassáridos, cassíidos, más los encontrados en el Tramo 2), escafópodos (dentálidos), así como fragmentos de própodos de crustáceos decápodos (callianásidos), cirrípedos balanomorfos y nanoplancton calcáreo. El contenido icnológico es monoespecífico y está representado por Ophiomorpha nodosa (Lundgren).

Entre la malacofauna encontrada destaca la presencia de varios taxones que son típicos del Plioceno, como Modiolus (M.) modiolus (Linneo), Tellina (Laciolina) incarnata (Linneo), Donax (Cuneus) venustus (Poli), Tugonia (T.) anatina (Gmelin), Palliolum (Lissochlamys) excisum (Bronn) y Gonimyrtea meneghinii (De Stefani y Pantanelli). Por otra parte, el análisis del nanoplancton calcáreo permite situar cronoestratigráficamente este tramo entre el F.O. de Gephyrocapsa spp. (-3,73 Ma) y los L.A.D. de Reticulofenestra umbilica (Gartner) y Sphenolithus spp. (-3,62 y -3,65 $\mathrm{Ma}$, respectivamente); es decir, en la biozona $\mathrm{CN} 11 \mathrm{~b}$ de Okada y Burky (1980, Zancliense superior; Muñiz Guinea, 1998). Esta datación permite establecer un hiato respecto al tramo anterior (Fig. 2) que abarcaría prácticamente todo el Messiniense y gran parte del Zancliense.

TRAMO 6: Arenas medias a muy gruesas, conglomerados y gravas rojizas, con matriz arenosa e intercalaciones, localmente, de arcillas blancas, en cuerpos lenticulares o inclinados, que pueden alcanzar extensiones de varias decenas de metros. Este conjunto litológico se dispone en aparente concordancia sobre el tramo anterior, aunque en ocasiones los conglomerados y gravas presentan una base suavemente erosiva. Hacia el norte y noroeste de la zona estudiada se disponen en discordancia erosiva y angular sobre los materiales del sustrato.

La potencia total del tramo oscila entre 2 y $6 \mathrm{~m}$. La parte inferior-media está constituida por unas arenas medias-muy gruesas, conglomerados y gravas con laminaciones inclinadas en dos sentidos: N130E con inclinación $15^{\circ}$ al SE y $\mathrm{N} 160 \mathrm{E}$ con inclinación $15^{\circ}$ al $\mathrm{NO}$, siendo su base erosiva. Son frecuentes los niveles arenosos y los conglomerados, que presentan en general cantos silíceos de mayor tamaño. La malacofauna fósil, en especial los bivalvos (que están mejor conservados) y el contenido icnológico, son similares a los del tramo inferior. Los cuerpos lenticulares de arcillas blancas tienen un espesor de 0,5 a 2,5 $\mathrm{m}$ y son más arenosos hacia techo, con pasadas microconglomeráticas de escala centimétrica $(<10 \mathrm{~cm})$. Presentan laminaciones paralelas horizontales e inclinadas de bajo ángulo y ripples de oscilación con direcciones $\mathrm{N} 110^{\circ} \mathrm{E}-\mathrm{N} 120^{\circ} \mathrm{E}$. La fauna es muy escasa, en general los bivalvos son de pequeño tamaño y sólo localmente aparecen niveles intensamente colonizados por Pholas sp. Estos niveles arcillosos se caracterizan por presentar restos abundantes de macroflora: Acer sp., Daphogenes sp., Laurophyllum aff. pseudoprinceps (Weyland y Kilpper), Platanus aceroides (Göppert), Pinus sp., Salix varians (Linneo), Zelkova sp. (Muñiz et al., 1999) con estados de conservación muy diferentes. La presencia de icnofósiles es baja, representada por los icnogéneros Palaeophycus, Thalassinoides, Gyrolithes y Skolithos. Hacia el oeste-noroeste estos niveles pasan a estar dominados totalmente por las arenas muy gruesas y gravas, masivas, que siguen manteniendo un contenido fosilífero muy alto. Los icnofósiles están representados por Ophiomorpha y Teichichnus.

TRAMO 7: Arenas finas-medias y limos arenosos, amarillentos, de 1 a $3 \mathrm{~m}$ de espesor. Los limos arenosos, localizados en la base, contienen una fauna fósil compuesta por bivalvos (árcidos, mitílidos, pectínidos, ostreidos, cardíidos, solénidos, tellínidos, donácidos, venéridos, hiatélidos), gasterópodos y escafópodos, aunque es más pobre que en los tramos inferiores. El contenido en icnotaxones es bajo, con sólo tres icnoespecies. Hacia el techo, las arenas finas-medias presentan una bioturbación muy intensa, caracterizada por la presencia de los icnogéneros Gyrolithes, Ophiomorpha y Teichichnus. La disposición de estos materiales es concordante, aparentemente, respecto a los del tramo inferior.

TRAMO 8: limos arenosos, blanco-amarillentos, de $12 \mathrm{~m}$ de espesor máximo, apoyados concordantemente sobre el tramo infrayacente. Presentan, hacia techo, intercalaciones de arenas medias con laminaciones paralelas horizontales y niveles conglomeráticos de espesor decimétrico. Al igual que en el tramo anterior, presentan costras ferruginosas de carácter diastémico. Otras estructuras sedimentarias también frecuentes son los moldes externos de crecimiento de cristales de sales, estructuras de escape de gases o fluidos, septarias y nódulos ferruginosos. El contenido faunístico se concentra en las superficies diastémicas, y su composición y estado de conservación es similar al presentado en el Tramo 2. Se encuentran bivalvos (nuculánidos, gliciméridos, mitílidos, pínnidos, pectínidos, ostreidos, lucínidos, astártidos, cardíidos, máctridos, solénidos, tellínidos, donácidos, solecúrtidos, venéridos, corbúlidos, hiatélidos), gasterópodos (turritélidos, capúlidos, nassáridos, natícidos, tónnidos, murícidos, olivínidos, cancelaríidos, cónidos, terebríidos), escafópodos (dentálidos), cirrípedos balanomorfos, própodos de crustáceos decápodos, restos de cetáceos y restos leñosos (en las costras ferruginosas superiores principalmente). Estas superficies, lateralmente, pueden dar paso a horizontes intensamente bioturbados y/o bioerosionados, representados por un alto numero de icnotaxones, aunque en menor número si se compara con el Tramo 2.

TRAMO 9: Arenas medias-gruesas, pardo-anaranjadas, 
limos y arenas caoliníticas, blancas. La potencia que presenta este tramo varía entre 1 y $4 \mathrm{~m}$. Las arenas mediasgruesas se localizan generalmente en la parte inferior y superior, tienen laminaciones paralelas horizontales e inclinadas en sentidos opuestos y de bajo ángulo, megaripples y ripples de corriente, así como canales de pequeña escala, aunque excepcionalmente se reconocen algunos de escala métrica. No se han encontrado restos de fauna, aunque Viguier (1974) cita en el kilómetro 3 de la carretera de Lepe a La Antilla una asociación de foraminíferos planctónicos pertenecientes a la Biozona de Globorotaria margaritae. El contenido icnológico está caracterizado por los icnogéneros Diplocraterion, Palaeophycus, Psilonichnus, Rosselia, Skolithos y Thalassinoides.

Los limos y arenas caoliníticas muestran mayor continuidad que estas arenas, sobre todo hacia el norte. Hacia el sur, se disponen de forma erosiva sobre las arenas glauconíticas del Mioceno Superior en el sector meridional, mientras que hacia el norte, lo hacen de forma aparentemente concordante sobre los limos arenosos, blanco-amarillentos del Plioceno Inferior y posiblemente Superior.

TRAMO 10: La sucesión estratigráfica culmina con un tramo de arenas medias-muy gruesas, blanco-rojizas, niveles discontinuos de gravas y conglomerados de cantos silíceos, de 3 a $11 \mathrm{~m}$ de espesor, cuya base es erosiva. Presentan laminación cruzada, recta o cóncava, de pequeña-mediana escala, formas canalizadas de base erosiva; así como estructuras asociadas con barras de arenas y gravas. La edad de este conjunto es Pleistoceno (Cáceres Puro, 1999), y se dispone mediante un contacto erosivo sobre los materiales del Mioceno y Plioceno, representando, en todo caso, una discordancia de tipo angular y erosiva.

\section{ICNOLOGÍA SISTEMÁTICA}

Icnogénero Spongeliomorpha Saporta, 1887

\section{Icnoespecie tipo: Spongeliomorpha iberica Saporta, 1887.}

\section{Diagnosis enmendada}

Madriguera con desarrollo horizontal dominante a ligeramente inclinado (entre $20^{\circ}$ y $30^{\circ}$ ) respecto al plano horizontal. Presentan ramificaciones en forma de "Y", según un ángulo aproximado de $60^{\circ}$ (ocasionalmente en forma de "T") y suelen terminar en extremos ciegos de morfología cónica o fusiforme. La sección transversal es circular a elíptica. La principal característica es la presencia de marcas fuertemente definidas en la pared exterior, que son longitudinales y transversales al eje principal de la madriguera y que convergen en el ápice de las ramificaciones. Estas marcas siguen unas trayectorias definidas, dibujando patrones más o menos regulares, subromboidales, sigmoidales o en forma de espiga. En ocasiones, entre las marcas que constituyen el entramado principal se encuentran otras más pequeñas que son perpendiculares a las primeras.

\section{Observaciones}

De Saporta (1887) definió Spongeliomorpha gracias

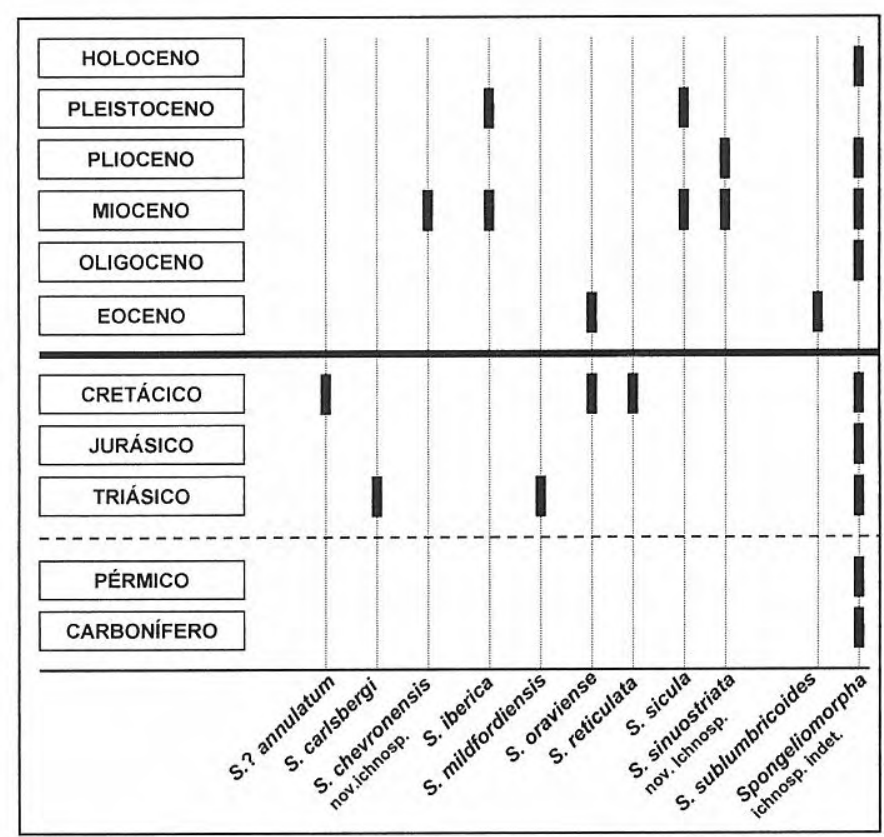

Figura 3. Distribución estratigráfica de las icnoespecies de Spongeliomorpha reconocidas hasta la fecha incluidas las descritas en el presente trabajo.

Stratigraphic distribution of the known ichnospecies of Spongeliomorpha.

al material recolectado por M. Vilanova en los depósitos marinos del Mioceno inferior de Alcoy (Alicante, España), proponiendo Spongeliomorpha iberica como la icnoespecie tipo. Fürsich (1973) estableció que las diferencias morfológicas entre los icnotaxones Spongeliomorpha, Thalassinoides y Ophiomorpha no justifican su distinción como pistas fósiles individuales, por lo que propuso considerar a las dos últimas como sinónimos de Spongeliomorpha. Sin embargo, Bromley y Frey (1974) y Marcinowski y Wierzowski (1975) creen que Spongeliomorpha debería ser considerada un nomen dubium. Por último, Calzada (1981) enmendó la diagnosis, tanto del icnogénero como de la icnoespecie tipo, usando el mismo material de la localidad citada anteriormente.

Spongeliomorpha es un icnotaxón cuyo registro estratigráfico es muy amplio (Carbonífero-Actualidad), aunque las diferentes icnoespecies abarcan intervalos de tiempo muy concretos (Muñiz Guinea, 1998; Muñiz et al., 1998b) (Fig. 3). Así, Spongeliomorpha? annulatum Kennedy aparece en el Cretácico superior del Sur de Inglaterra (Kennedy, 1967); S. sublumbricoides (Azpeitia Moros), en el Cretácico de Zumaya (Vizcaya, España) (Azpeitia Moros, 1933, en Uchman, 1998); S. oraviense (Książkiewicz), en el Eoceno de los Carpatos polacos (Książkiewicz, 1977, en Uchman, 1998); S. iberica de Saporta, en el Mioceno inferior de Alcoy (Alicante, España) (Calzada, 1981) y S. sicula D'Alessandro y Bromley, en el Mioceno superior de Lepe (Huelva, España) y Praia Verde (Algarve, Portugal) (Muñiz et al., 1998b); así como en el Pleistoceno Inferior de Sicilia en Italia (D’Alessandro y Bromley, 1995). Todas ellas están 


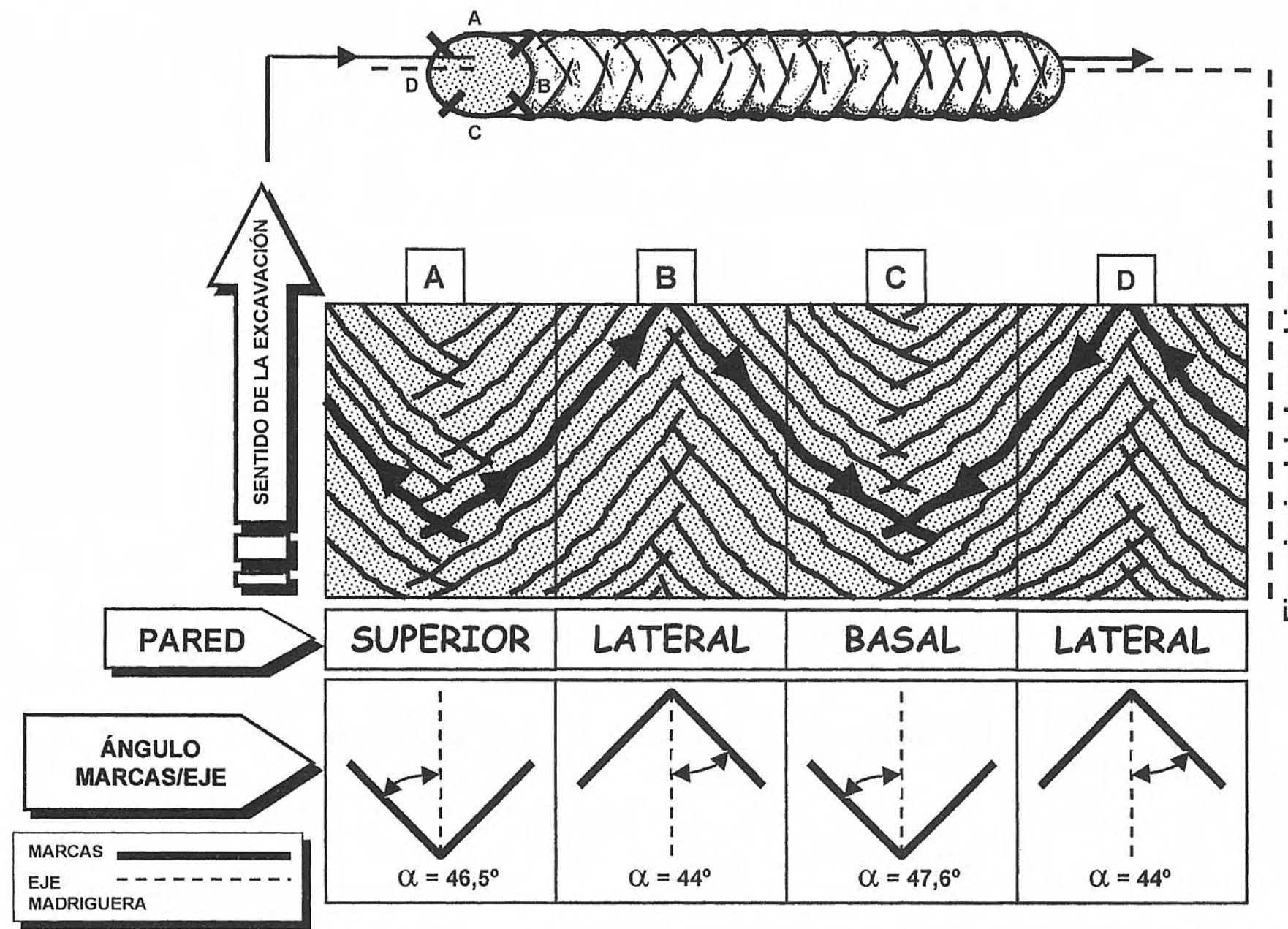

Figura 4. Reconstrucción del patrón ornamental que configuran las marcas en las paredes exteriores de Spongeliomorpha chevronensis nov. ichnosp. a partir de la relación angular de estas con el eje principal de la madriguera ( $\alpha$ está dado en valor medio). Se indica a su vez el sentido de avance de la excavación (explicación en el texto).

Reconstruction of the ornamental pattern developed by the ridges on the external walls of Spongeliomorpha chevronensis nov. ichnosp. from the angular relation between these ridges and the main axis of the burrow. The sense of the movement in the burrowing is showing, too (see text for further information).

relacionadas con ambientes estrictamente marinos. Uchman (1998) puso en sinonimia Halymenidium Schimper, 1869 con Spongeliomorpha y pasó a considerar Halymenidium sublumbricoides Azpeitia Moros, 1933 y H. oraviense Książkiewicz, 1977, como Spongeliomorpha sublumbricoides y Spongeliomorpha oraviense, respectivamente.

Por otra parte, en relación con ambientes acuáticos no marinos, se han descrito Spongeliomorpha carlsbergi Ekdale et al., 1984, para el Triásico de Groenlandia y $S$. mildfordiensis Metz, 1993, para el Triásico de Nueva Jersey (EEUU). Spongeliomorpha de asignación icnoespecífica indeterminada y relacionadas con ambientes marinos se han citado para el Pérmico inferior de la Cuenca de Sydney en Australia (Eyles et al., 1998), Triásico, Jurásico y Cretácico de Alemania (Weigelt, 1929), Triásico medio del NE de la Columbia Británica en Canadá (Zonneveld et al., 1998), Cretácico de Francia (Lessertisseur, 1955), Cretácico Superior (Turoniense) del Sur de Inglaterra (Gale, 1996) y de la Supersecuencia Orfento en Maiella, Italia (Mutti et al., 1996), Paleógeno inferior del NE de Italia (Ghibaudo et al., 1996), Mioceno y Plioceno de Lepe (Huelva, España) (Muñiz et al., 1995; Muñiz Guinea, 1998; Muñiz et al., 1998a; Muñiz y Mayoral, 1998) y Holoceno de Asturias, en España (Flor et al., 1979). Igualmente, en ambientes acuáticos no marinos, se han referido para el Carbonífero de New Brunswick en Canadá (Pickerill, 1992) y recientemente, Uchman y Alvaro (1999, 2000), en depósitos lacustres miocenos de la Depresión de Calatayud (Zaragoza)Teruel, NE de España.

Desde el punto de vista etológico, Spongeliomorpha se considera como una estructura de búsqueda de alimento (Calzada, 1981), aunque también se ha interpretado de manera secundaria como una construcción con fines de morada temporal (Muñiz et al., 1995; Muñiz Guinea, 1998; Muñiz et al., 1998b) y en el caso especial de Spongeliomorpha sicula, como una estructura de granja/trampa (D'Alessandro y Bromley, 1995; Muñiz et al., 1998b). Spongeliomorpha es frecuente en los sistemas intergradacionales de pistas junto con Ophiomorpha o Thalassinoides (Kennedy, 


\begin{tabular}{|c|c|c|c|c|c|}
\hline \multirow{2}{*}{$\begin{array}{c}\text { ICNOESPECIES } \\
\text { Spongeliomorpha } \\
\text { chevronensis } \\
\text { nov. ichnosp. } \\
\text { (en este trabajo) }\end{array}$} & \multirow{2}{*}{$\begin{array}{c}\text { PATRÓN DE LAS } \\
\text { MARCAS } \\
\text { REGULAR } \\
\text { (en forma de espiga) }\end{array}$} & \multicolumn{2}{|r|}{$\begin{array}{c}\text { ÁNGULO } \\
\text { Marcas/Eje madriguera }\end{array}$} & ESQUEMA & $\begin{array}{l}\text { FORMA DE LA } \\
\text { RAMIFICACIÓN }\end{array}$ \\
\hline & & $\begin{array}{l}P \\
A \\
R \\
E \\
D\end{array}$ & $\begin{array}{l}\text { Superior: } 46,5^{\circ} \\
\text { Inferior: } 47,6^{\circ} \\
\text { Laterales: } 44^{\circ} \\
\text { (valor medio) }\end{array}$ & & $\gamma_{\alpha=58^{\circ}-72^{\circ}}^{\alpha}$ \\
\hline $\begin{array}{c}\text { Spongeliomorpha } \\
\text { carlsbergi } \\
\text { Ekdale et al. } 1984\end{array}$ & IRREGULAR & & $70^{\circ}-90^{\circ}$ & & \\
\hline $\begin{array}{c}\text { Spongeliomorpha } \\
\text { mildfordiensis } \\
\text { Metz, } 1993\end{array}$ & IRREGULAR & & $\begin{array}{c}58^{\circ}-72^{\circ} \\
\text { (según Metz, 1993) }\end{array}$ & & $\alpha=63^{\circ}($ Metz, 1993) \\
\hline $\begin{array}{c}\text { Spongeliomorpha } \\
\text { oraviense } \\
\text { Ksiazkiewicz, } 1977\end{array}$ & IRREGULAR & & $\begin{array}{c}30^{\circ}-65^{\circ} \\
\text { (comunicación personal } \\
\text { A. Uchman) }\end{array}$ & & ¿? \\
\hline $\begin{array}{l}\text { Spongeliomorpha } \\
\text { sublumbricoides } \\
\text { Azpeitia Moros, } 1933\end{array}$ & IRREGULAR & & $\begin{array}{c}35^{\circ}-85^{\circ} \\
\text { (comunicación personal } \\
\text { A. Uchman) }\end{array}$ & 1) $\int_{i}^{1}$ & \\
\hline $\begin{array}{l}\text { Spongeliomorpha } \\
\text { ichnosp. indet } \\
\text { (en este trabajo) }\end{array}$ & IRREGULAR & & $30^{\circ}-87^{\circ}$ & & NO OBSERVADA \\
\hline
\end{tabular}

Tabla 1. Principales características de las icnoespecies de Spongeliomorpha que tienen las marcas transversales respecto al eje de la madriguera (Grupo 3, ver texto). El esquema de Spongeliomorpha carlsbergi se ha conseguido a partir de la fig. 12, p. 58 del trabajo de Bromley y Asgaard (1979), el de S. mildfordiensis, de la fig. 2, p. 260 en Metz (1993), los de S. oraviense y S. sublumbricoides, de las figs. 1p y 1q, p. 284, ambas en Książkiewicz (1977).

Main characteristics of the ichnospecies of Spongeliomorpha showing transversal ridges respect to the main axis of the burrow (Group 3, see text). The schematic drawing of Spongeliomorpha carlsbergi is based on the fig. 12, p. 58 in Bromley and Aasgard (1979), the drawing of S. mildfordiensis, from the fig. 2, p. 260 in Metz (1993), and the sketchs of $\mathrm{S}$. oraviensis and $\mathrm{S}$. sublumbricoides on the figs. 1p and 1q, p. 284 both in Ksiązkiewicz. (1977).

1967; Kennedy y McDougall, 1969; Bromley y Frey, 1974) y Teichichnus-Phycodes (Muñiz et al., 1995; Muñiz Guinea, 1998). Las interpretaciones sobre los posibles organismos productores son numerosas y diversas. De Saporta (1887) y de Laubenfels (1955) lo atribuyen a esponjas; Fuchs (1895) a posibles anélidos tipo Terebella; Bosca (1917) y Darder (1945) a algas; mientras que Ksiđøkiewicz (1961, 1977) atribuye su origen a una acción de posibles holotúridos, ophiúridos o sipuncúlidos. La interpretación más aceptada sobre su génesis estaría relacionada con la actividad excavadora de crustáceos decápodos (Kennedy, 1967; D’Alessandro y Bromley, 1995; Muñiz et al., 1995; Muñiz Guinea, 1998; Muñiz et al., 1998b, entre otros). En esta línea, Flor et al. (1979) citan estructuras similares relacionadas con callianásidos en depósitos marinos actuales. Por otro lado, Bromley y Asgaard (1979), Ekdale et al. (1984) y Metz (1993, 1995) relacionan algunas de las icnoespecies, en concreto Spongeliomorpha carlsbergi y S. mildfordiensis, con la actividad vital de larvas de insectos acuáticos no marinos.

Los ejemplares recolectados se hallan depositados en el Departamento de Geodinámica y Paleontología de la Universidad de Huelva, excepto el ejemplar LE14/S6, que se halla en el Museo Paleontológico del Departamento de Geología de la Universidad de Zaragoza. Todos los ejemplares se conservan como relieves completos y están ferruginizados.

\section{Spongeliomorpha chevronensis nov. ichnosp. Figs. 4, 5a-d; Tab. 1}

Material tipo: treinta ejemplares recolectados. Holotipo: LE14/Py45. Paratipos: LE5/Py 1 a LE5/Py3, LE7/Py2, LE9/Py 1 y 2, LE10/Py3, LE11/Py 13, LE13/Py 2 y 3, LE14/Py3 y LE14/Py46 a LE14/Py64.

Localidad tipo: Lepe (Huelva, SO de España).

Estrato tipo: Tramo 2 de la sección litoestratigráfica antes mencionada (Fig. 2).

Edad: Mioceno Superior (Tortoniense superior).

Origen del nombre: del francés chevron= forma de espiga. Relativo al patrón que presentan las marcas de rastrillaje.

\section{Diagnosis}

Spongeliomorpha recta a suavemente curvada en planta; caracterizada por una fuerte ornamentación en toda la pared exterior, con marcas de rastrillaje oblicuas respecto al eje principal de la galería, configurando un patrón en forma de espiga. Las ramificaciones, poco frecuentes, son en forma de "Y" y las terminaciones cónicas.

Spongeliomorpha straight to gently curved, with the external wall runned by long, oblique, ear-shaped ridges regularly distributed respect to the main axis of the 


\begin{tabular}{|c|c|c|c|c|}
\hline ICNOESPECIES & $\begin{array}{l}\text { PATRÓN DE LAS } \\
\text { MARCAS }\end{array}$ & $\begin{array}{l}\text { MORFOLOGIAA DEL } \\
\text { ENREJADO }\end{array}$ & ESQUEMA & $\begin{array}{l}\text { FORMA DE LA } \\
\text { RAMIFICACIÓN }\end{array}$ \\
\hline $\begin{array}{l}\text { Spongeliomorpha? } \\
\text { annulatum } \\
\text { Kennedy, } 1967\end{array}$ & IRREGULAR & $\begin{array}{c}\text { MARCAS } \\
\text { LONGITUDINALES }\end{array}$ & $=-12=$ & $\varliminf_{\alpha=35^{\circ}-40^{\circ}}^{\alpha}$ \\
\hline $\begin{array}{l}\text { Spongeliomorpha sicula } \\
\text { D'Alessandro \& } \\
\text { Bromley, } 1995\end{array}$ & REGULAR & $\begin{array}{c}\text { MARCAS } \\
\text { SUBLONGITUDINALES }\end{array}$ & CÁMARA E & $\bigcap_{\alpha=45^{\circ}-70^{\circ}}^{\alpha}$ \\
\hline
\end{tabular}

Tabla 2. Principales características de las icnoespecies de Spongeliomorpha que tienen las marcas más o menos subparalelas respecto al eje principal (Grupo 2, ver texto). El esquema de Spongeliomorpha? annulatum se ha obtenido a partir de la fig. 7, lám. 7 en Kennedy (1967) y el de $S$. sicula de las figuras. 4 y 7.2 de las páginas 395 y 396 respectivamente, en D'Alessandro y Bromley (1995).

Main characteristics of the ichnospecies of Spongeliomorpha showing subparallel ridges on the main axis of the burrow (Group 2, see text). The sketch of Spongeliomorpha? annulatum have been obtained from the fig. 7, pl. 7 in Kennedy (1967), and the drawing of S. sicula from the figs. 4 and 7.2 in D'Alessandro and Bromley (1995).

burrow. The $Y$-shaped branching are rare and the burrows end in conical, blind points.

\section{Descripción}

Madrigueras horizontales a ligeramente inclinadas, con trayectorias rectas a curvadas. Las ramificaciones son poco frecuentes, su desarrollo es corto, en forma de "Y" (ángulo entre $58^{\circ}$ y $62^{\circ}$ ) y sus extremos son ciegos, de forma cónica o fusiforme (Fig. 5b,d).

La pared exterior, débilmente revestida, presenta una fuerte ornamentación constituida por marcas de rastrillaje (1 a $2 \mathrm{~mm}$ de grosor), largas, ligeramente arqueadas, que forman ángulos agudos entre sí y respecto al eje principal de la galería, lo que ocasiona un patrón muy característico con una morfología general en forma de espiga (Fig. 5a, c). El ángulo que forman las marcas con el eje de la madriguera varía en función del sector que éstas ocupen. Así, cuando aparecen en la parte superior de la madriguera, su valor es de $42^{\circ}$ a $47^{\circ}\left(46,5^{\circ}\right.$ en promedio, Fig. $\left.4 \mathrm{~A}\right)$, si lo hacen en la inferior, es de $46^{\circ}$ a $48^{\circ}\left(47,6^{\circ}\right.$ de media, Fig. $\left.4 \mathrm{~B}\right)$ y si se presentan en las zonas laterales, de $40^{\circ}$ a $45^{\circ}$ (media de $44^{\circ}$, Fig. 4C-D).

La sección transversal es circular a subcircular, con un diámetro de 1,4 a $3,5 \mathrm{~cm}$ (valor medio de $2 \mathrm{~cm}$ ). La longitud máxima observada es de $14 \mathrm{~cm}$. El relleno pasivo, en pocas ocasiones zonado, es de litología semejante a la del sedimento circundante. Suelen formar parte de sistemas intergradacionales más o menos complejos donde la estructura proximal está formada por Spongeliomorpha, la de transición por Teichichnus y la distal por Phycodes (Muñiz et al., 1995).

\section{Spongeliomorpha sicula D’Alessandro y Bromley, 1995 \\ Fig. 5 e-h; Tab. 2}

Material estudiado: Se han reconocido dieciséis ejemplares: LE11/Ss1 a LE11/Ss 16. Igualmente se han considerado las observaciones realizadas en los ejemplares conservados in situ en los afloramientos.

\section{Diagnosis}

Spongeliomorpha con cámaras ovoidales, dispuestas verticalmente hacia arriba, localizadas sobre los puntos de ramificación de las galerías. Las galerías se disponen a diferentes niveles de profundidad y se conectan por fustes bien desarrollados. Las paredes de las cámaras y galerías están ornamentadas por crestas y surcos sublongitudinales que se cruzan entre sí (D’Alessandro y Bromley, 1995).

Figura 5. a-d. Spongeliomorpha chevronensis nov. ichnosp. a. Holotipo: LE14/Py45, x1,4 vista inferior. Obsérvese las marcas en forma de espiga (chevron). b. Paratipo: LE14/Py64, x0,8 vista inferior en la que se observa la ramificación en forma de "Y" y la terminación cónica. c. Paratipo: LE9/Py1, x1,2 vista lateral. d. Paratipo: LE14/Py63, x1,25). Detalle de la terminación cónica de una ramificación. e-h. Spongeliomorpha sicula D'Alessandro y Bromley. e. Ejemplar LE11/Ss1, x1,18, vista lateral. f. LE11/Ss16, x1,15, vista superior. g. LE11/Ss15, x1,32, vista lateral. h. LE11/Ss8, x1,72, vista superior. Obsérvese como las marcas convergen en el ápice de la cámara.

a-d. Spongeliomorpha chevronensis nov. ichnosp. a. Holotype: LE14/Py45, x1.4, basal view. Note the chevron-shaped ridges. $b$. Paratype: LE14/Py64, x0.8; basal view. Note the Y-shaped branching and the conical, blind end. $c$. Paratype: LE9/Py1, x1.2, lateral view. d. Paratype: LE14/Py63, x1.25. Detail of a conical end. $\boldsymbol{e}$-h. Spongeliomorpha sicula D'Alessandro and Bromley. e. Specimen LE11/Ss1, x 1.18, lateral view. f. LE11/Ss16, x1.15, upper view. g. LE11/Ss15, x1.32, lateral view. h. LE11/Ss8, x1.72, upper view. Note the ridges converging to the apex of the chamber. 
a

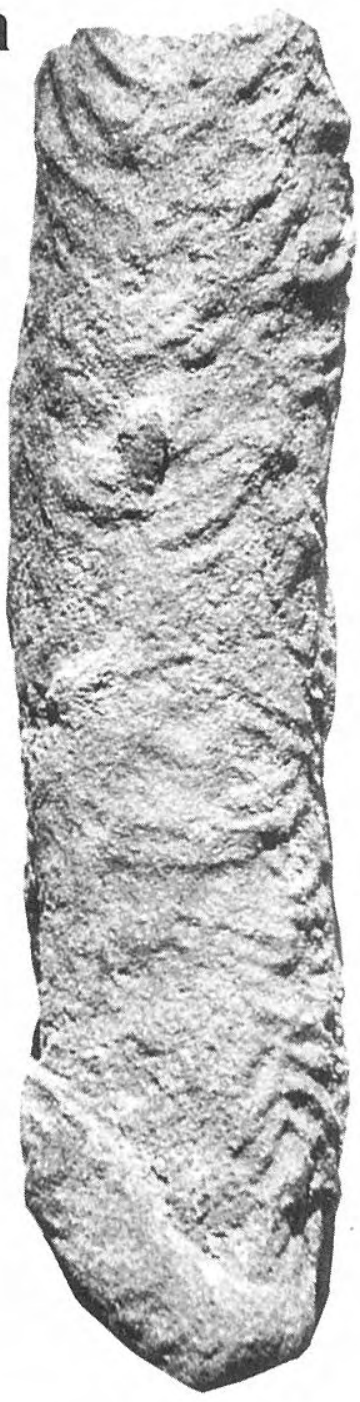

e
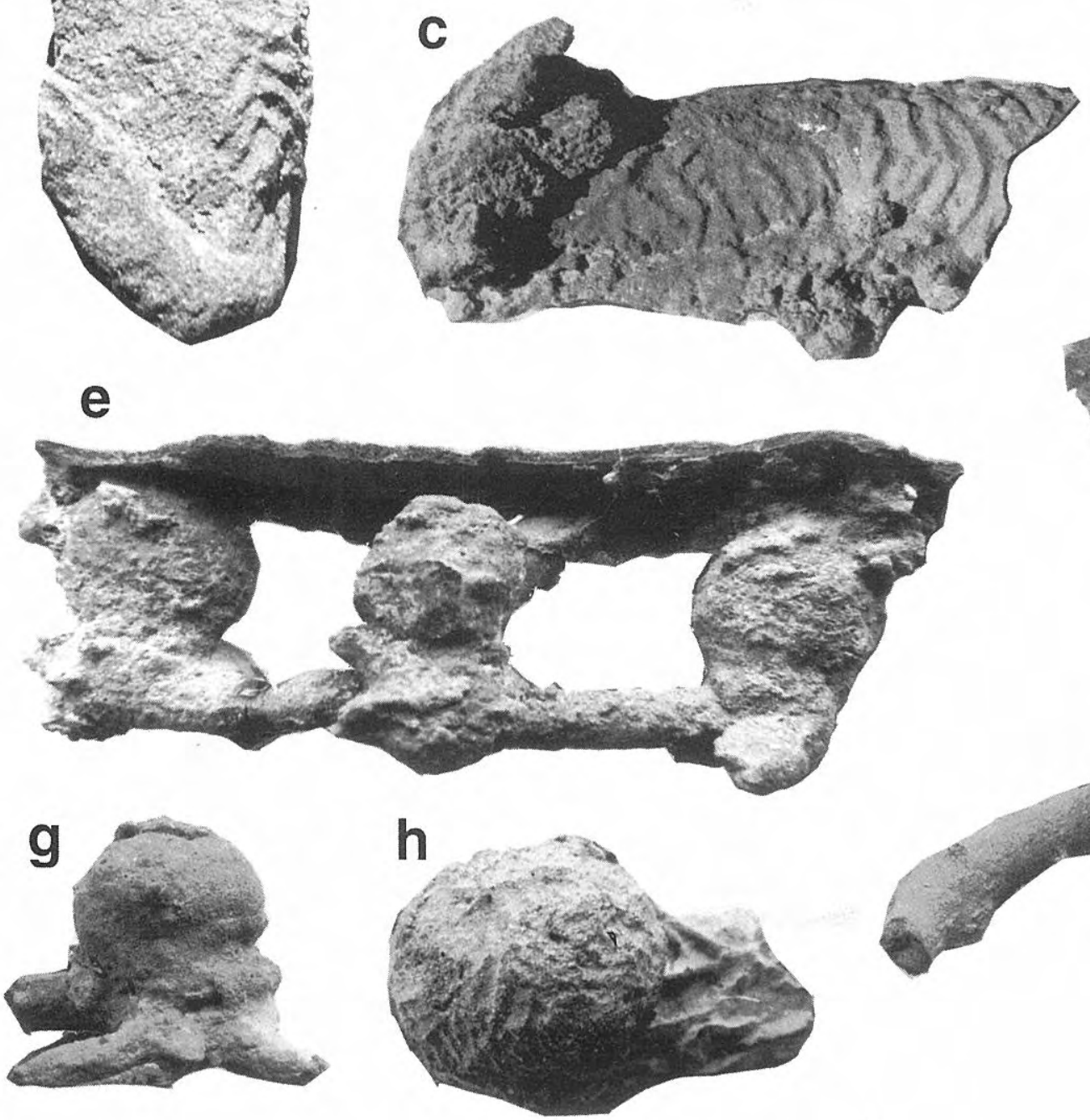
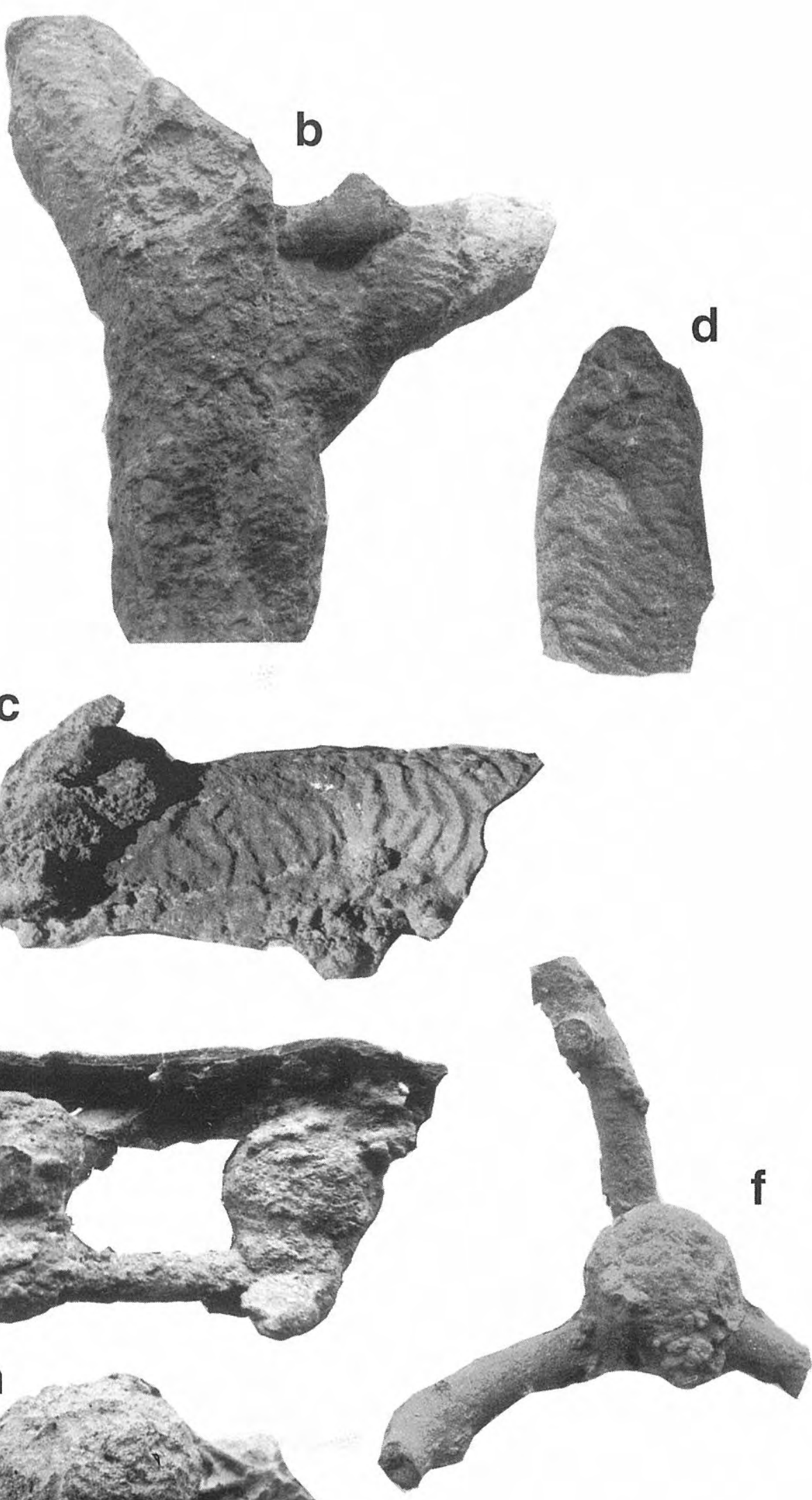
A

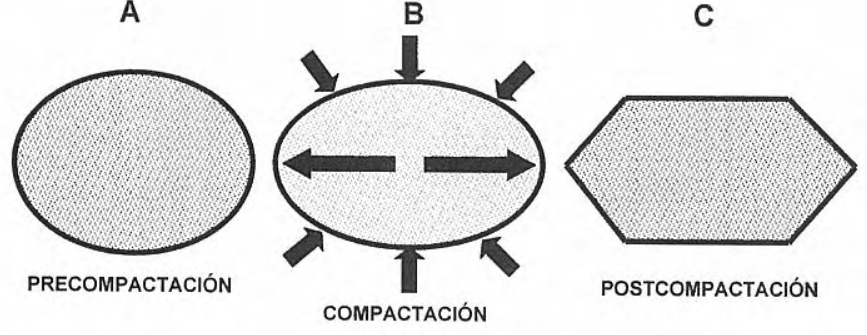

Figura 6. Reconstrucción de las secciones transversales de Spongeliomorpha sinuostriata nov. ichnosp. (explicación en el texto).

Reconstruction of the transversal sections of Spongeliomorpha sinuostriata nov. ichnosp. (see text for further explanation).

\section{Descripción}

Entramados simples, que pueden ocupar varios niveles de profundización, aunque siempre muy someros (no más de 10 $\mathrm{cm}$ ) compuestos por galerías de sección subcircular a elíptica (la altura varía entre 0,5 y $0,9 \mathrm{~cm}$ y la anchura entre 0,7 y 1 $\mathrm{cm}$ ), con una longitud máxima observada de $9 \mathrm{~cm}$ y un revestimiento muy fino. Estas galerías presentan ramificaciones, que suelen ser en forma de "Y" (ángulo entre $50^{\circ}$ y $70^{\circ}$ ). No se han observado sus terminaciones. En los puntos de ramificación se localizan las típicas cámaras (Fig. 5f) de aspecto globoso, de contorno redondeado-subovoidal, que se disponen en la vertical y hacia arriba, con un diámetro que varía entre 1,3 y $2,5 \mathrm{~cm}$ y un altura máxima de $2 \mathrm{~cm}$.

Las paredes de todo el sistema anterior están fuertemente ornamentadas en su parte exterior por marcas que configuran un patrón más o menos regular. En las galerías se presentan cruzadas entre sí, con una ligera inclinación respecto a su eje principal. La ornamentación es más acusada en los flancos y techos, siendo menor en las paredes inferiores. En las cámaras globosas, las marcas tienden a converger hacia el ápice (Fig. 5h). El relleno (pasivo en todos los casos) es algo más grueso que el del sedimento circundante y son frecuentes las yuxtaposiciones con otras pistas.

\section{Spongeliomorpha sinuostriata nov. ichnosp.} Figs. 6, 7a-d, f,g; Tab. 3
Material tipo: ciento ocho ejemplares recolectados. Holotipo: LE6/S8. Paratipos: LE1/S1 a LE1/S3, LE3/S1, LE4/S1 y 2, LE5/S 1 a LE5/S6, LE6/S1 a LE6/S7 y de LE6/S9 a LE6/S28, LE7/S1 y 2, LE9/S1 a LE9/S30, LE10/S1 y 2, LE11/S1 a LE11/S26, LE13/S1 y 2, LE14/S1 a LE14/S5 y LE16/S1 a LE16/S5.

Localidad tipo: Lepe (Huelva, SO de España).

Estrato tipo: Tramos 2 y 8 de la sección litoestratigráfica global (Fig. 2).

Edad: Mioceno Superior (Tortoniense superiorMessiniense) y Plioceno Inferior (Zancliense superior).

Origen del nombre: denominación latina compuesta por los términos sinuoso (sinuosus - $a$-um) y estriado (striatus $-a$ um), sinuestriado. Referente a la trayectoria sinuosa que presentan las marcas de rastrillaje.

\section{Diagnosis}

Spongeliomorpha de sección transversal, subelipsoidal, con ramificaciones cortas, aparentemente en forma de "Y" y terminadas en formas cónicas. La pared externa está ornamentada por un sistema de marcas, con trayectoria sinuosa respecto al eje principal de la galería y yuxtapuestas entre sí, que dan lugar a un enrejado sigmoidal.

Spongeliomorpha subelliptical in cross-section with the external wall runned by long and sinuous ridges subparallel to the main axis of the burrow and irregulary scattered along it. Ridges are juxtapose producing a sigmoidal network. The burrow presents a short scarce apparently $Y$-shaped branching with conical-like ends.

\section{Descripción}

Madrigueras horizontales a inclinadas $\left(27,6^{\circ}\right.$ de media respecto a la horizontal), de trayectoria rectilínea. Las ramificaciones son poco frecuentes; cuando aparecen, su longitud es corta y lo hacen en forma de "Y" (ángulo entre $55^{\circ}$ y $63^{\circ}$ ), acaban en extremos ciegos, de forma cónica o fusiforme (Fig. 7f, g).

La pared exterior está fuertemente ornamentada por

Figura 7. a-d, f-g. Spongeliomorpha sinuostriata nov. ichnosp. a. Holotipo: LE6/S8, X 1,09, vista lateral. Obsérvese la trayectoria sinuosa que presentan las marcas. b. Paratipo: LE9/S30, X 1,37, vista lateral. c. Paratipo: LE9/S29, X1,26, vista lateral. d. Paratipo: LE6/S10, X 1,21) vista superior. f-g. Detalle de la terminación cónica de las ramificaciones, f: Paratipo, LE9/S1, X1,08; g: Paratipo, LE9/S2, X 1,3. e. Ejemplar de Teichichnus ichnosp. indet. (Le6/TES1, X 1,1), vista lateral. h-j. Spongeliomorpha ichnosp. indet. h. Ejemplar LE11/Si1, X 1,5, vista lateral. Obsérvese el detalle de las marcas oblícuas respecto al eje principal de la madriguera. i. LE11/Si2, X 1,14, vista superior. j. LE9/Si1, X 1,27, vista inferior. Obsérvese en i y j el detalle de las marcas dispuestas casi perpendiculares respecto del eje principal de la madriguera.

$\boldsymbol{a}-\boldsymbol{d}, \boldsymbol{f}$-g. Spongeliomorpha sinuostriata nov. ichnosp. a. Holotype: LE6/S8, x 1.09, lateral view. Note the sinuous pathway of the ridges. b. Paratype: LE9/S30, x 1.37, lateral view. c. Paratype: LE9/529, X 1.26, lateral view. $d$. Paratype: LE6/S10, x 1.21, upper view. f-g. Detail of the conical end of the burrows; f: Paratype, LE9/S1, x 1.08; g: Paratype, LE9/S2, x 1.3. e. Specimen of Teichichnus ichnosp. indet., LE6/TES1, x 1.1, lateral view. $\boldsymbol{h}$-j. Spongeliomorpha ichnosp. indet. h. Specimen LE11/Si1, $x$ 1.5, lateral view. Close-up view of the oblique ridges in relation to the main axis of the burrow. $i$. Specimen LE11/Si2, x 1.14, upper view; $j$. LE9/Si1, x 1.27, basal view. Note in both specimens the ridges distributed perpendicularly respect to the main axis of the burrow. 

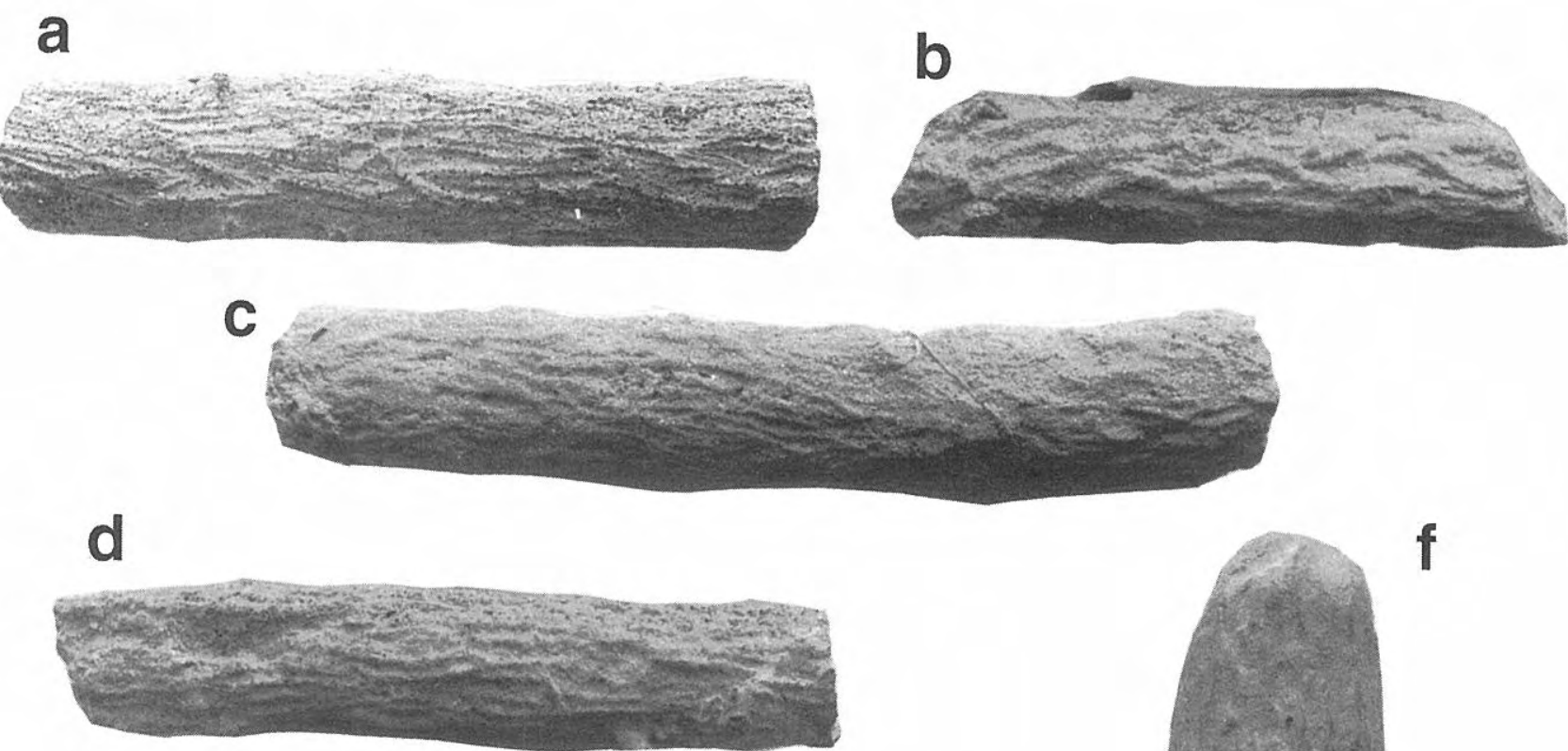

e
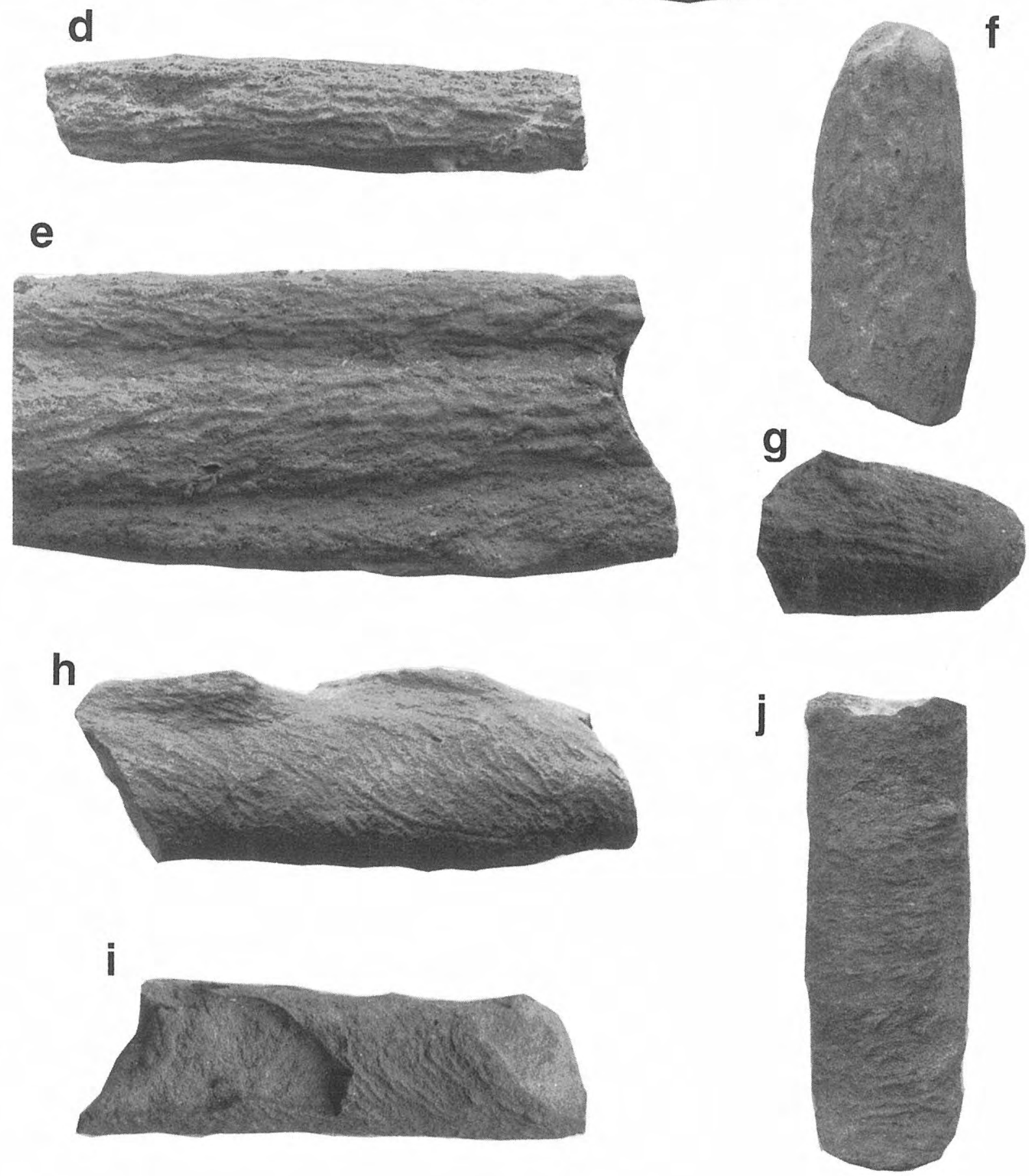


\begin{tabular}{|c|c|c|c|c|}
\hline ICNOESPECIES & $\begin{array}{l}\text { PATRÓN DE LAS } \\
\text { MARCAS }\end{array}$ & $\begin{array}{l}\text { MORFOLOGÍA DEL } \\
\text { ENREJADO }\end{array}$ & ESQUEMA & $\begin{array}{l}\text { FORMA DE LA } \\
\text { RAMIFICACIÓN }\end{array}$ \\
\hline $\begin{array}{c}\text { Spongeliomorpha } \\
\text { sinuostriata } \\
\text { nov. ichnosp. } \\
\text { (en este trabajo) }\end{array}$ & IRREGULAR & SIGMOIDAL & $\frac{15}{210}$ & $\prod_{\alpha=55^{\circ}-63^{\circ}}^{\alpha}$ \\
\hline $\begin{array}{c}\text { Spongeliomorpha } \\
\text { iberica } \\
\text { Calzada, } 1981 \\
\end{array}$ & REGULAR & SUBROMBOIDAL & 2018 & $\prod_{\alpha=60^{\circ}}^{\alpha}$ \\
\hline $\begin{array}{l}\text { Spongeliomorpha } \\
\text { reticulata } \\
\text { Chiplonkar \& Ghare, } 1993\end{array}$ & IRREGULAR & SUBCUADRANGULAR & & ¿? \\
\hline
\end{tabular}

Tabla 3. Principales características de las icnoespecies de Spongeliomorpha que presentan las marcas con un modelo en enrejado (Grupo 1, ver texto). El esquema de Spongeliomorpha iberica está realizado a partir de la fig. 4, lám II, p. 193 en Calzada (1981) y el de S. reticulata, de la fig. 3, p.77 en Chimplonkar y Ghare (1993).

Main characteristics of the ichnospecies of Spongeliomorpha showing a network pattern produced by juxtaposition of ridges (Group 1, see text). The drawing of Spongeliomorpha iberica have been realized from the fig. 4, pl. II, p. 193 in Calzada (1981) and the sketch of S. reticulata on the fig. 3, p. 77 in Chiplonkar and Ghare (1993).

marcas que configuran un patrón más o menos complejo. Generalmente, se orientan en sentido longitudinal respecto al eje principal de la madriguera, son de trazado sinuoso más o menos constante y cuando se intersectan unas con otras, dibujan un enrejado irregular de formas sigmoidales (Fig. $7 a-d)$. Suelen converger en el ápice de las ramificaciones cónicas. La anchura de estas marcas varía de $<1 \mathrm{~mm}$ a 2,2 $\mathrm{mm}$, con un valor medio de $1,15 \mathrm{~mm}$ (en sesenta y cuatro medidas efectuadas).

La sección transversal de las madrigueras varía de subelipsoidal a hexagonal-subhexagonal; la anchura y la altura oscilan entre $0,9-2,5 \mathrm{~cm}$ y $0,7-2,4 \mathrm{~cm}$, respectivamente (ambos parámetros medidos en cuarenta y nueve ejemplares de sección subelipsoidal). El revestimiento, muy fino, tiene un espesor medio de $1,25 \mathrm{~mm}$, la longitud máxima de los ejemplares estudiados es de $15 \mathrm{~cm}$.

El relleno (pasivo en todos los casos) es más grueso que el sedimento circundante, siendo frecuente observar, tanto ligados al revestimiento como al interior, granos de glauconita y chamosita (exclusivo para los ejemplares del Tramo 2, ver Fig. 2); así como restos de microfauna. Por lo general, se encuentran aisladas o formando parte de sistemas intergradacionales incompletos en conexión con Teichichnus (Fig. 7e).

\section{Spongeliomorpha ichnosp. indet.} Fig. 7h-j; Tab. 1

Material estudiado: se han recolectado cuatro ejemplares: LE11/Si1, LE11/Si2, LE9/Si1 y LE14/Si1.

\section{Descripción}

Spongeliomorpha que presenta la pared exterior con marcas de rastrillaje oblicuas (30 máximo) (Fig. 7 h) a casi perpendiculares (Fig. 7i, j) respecto al eje principal de la madriguera (ver Tabla 1), muy finas $(<1 \mathrm{~mm})$, ordenadas irregularmente, con una trayectoria recta a ligeramente ondulada. Las yuxtaposiciones son poco frecuentes.
La sección transversal varía entre subelipsoidal a circular, con una anchura de $2 \mathrm{~cm}$ y una altura de $1,7 \mathrm{~cm}$. El revestimiento es muy delgado $(<1 \mathrm{~mm})$ y la longitud máxima observada en los ejemplares recolectados es de $8 \mathrm{~cm}$. No se observan las ramificaciones. El relleno (arenas finas) en todos los casos es pasivo, más grueso que el sedimento circundante (limos arenosos).

\section{DISCUSIÓN}

Hasta la fecha se pueden establecer tres agrupaciones morfotípicas a partir de las icnoespecies de Spongeliomorpha si consideramos el criterio icnotaxobásico que las caracteriza, es decir, la presencia y disposición de las marcas de rastrillaje respecto al eje principal de la madriguera.

Así, se diferencia un primer grupo donde las marcas se cruzan entre sí para dar lugar a un enrejado subromboidal, sigmoidal o subcuadrangular. Es el caso de las icnoespecies (Tabla 3): Spongeliomorpha iberica, S. reticulata Chiplonkar y Gare, 1975 y S. sinuostriata nov. ichnosp. Hay un segundo grupo donde las marcas raramente se cruzan y son más o menos longitudinales al eje. A este grupo pertenecen las icnoespecies (Tabla 2): S.? annulatum Kennedy, 1967 y S. sicula D'Alessandro y Bromley, 1995. Y por último, existe un tercer grupo que tiene las marcas dispuestas transversalmente respecto al eje, según una relación angular que oscila entre $30^{\circ}-35^{\circ}$ a $90^{\circ}$, y que ocasionalmente pueden llegar a cruzarse, pero en ningún caso formando un claro enrejado como en las anteriores icnoespecies. En este grupo se incluyen (Tabla 1): Spongeliomorpha carlsbergi, $S$, mildfordiensis, $S$. oraviense, S. sublumbricoides, así como S. chevronensis nov. ichnosp. y Spongeliomorpha. ichnosp. indet. descrita en este trabajo.

Entre las madrigueras que pertenecen al primer grupo (ver Tabla 3), podemos establecer las siguientes comparaciones a nivel icnoespecífico: Spongeliomorpha 
sinuostriata nov. ichnosp. difiere de las otras icnoespecies, en especial de $S$. iberica y $S$. reticulata, por presentar un patrón ornamental diferente, caracterizado por un enrejado sigmoidal originado por el cruce de marcas sinuosas. En el segundo grupo, S. sicula se diferencia por la trayectoria recta de las marcas, su trazado regular y sobre todo, porque es la única icnoespecie que presenta cámaras ovoidales en conexión. Respecto a las del tercer grupo (ver Tabla 1), Spongeliomorpha chevronensis nov. ichnosp. se distingue principalmente del resto de icnoespecies porque sus marcas presentan una relación angular muy regular, mientras que en las restantes esta relación varía considerablemente. Así, S. carlsbergi, S. mildfordiensis, S. oraviense, S. sublumbricoides y Spongeliomorpha ichnosp. indet. (en este trabajo) exhiben en todos los casos una relación angular mucho más irregular (los ángulos oscilan entre $30^{\circ}$ y $90^{\circ}$ ). Referente a Spongeliomorpha ichnosp. indet., no se encuentran parecidos con las icnoespecies que integran este grupo. El número de ejemplares, el estado de conservación y el alto grado de fragmentación, imposibilitan de momento una comparación a estos niveles.

\section{POSIBLES ORGANISMOS CONSTRUCTORES E INTERPRETACIONES PALEOETOLÓGICAS}

Datos precedentes relacionan estas madrigueras con organismos sedimentívoros y suspensívoros, posiblemente crustáceos decápodos macruros, callianásidos o talasínidos. En nuestra opinión, para las icnoespecies Spongeliomorpha chevronensis nov. ichnosp., $S$. sinuostriata nov. ichnosp. y Spongeliomorpha ichnosp. indet., la presencia de las numerosas marcas de rastrillaje en las paredes exteriores sugiere una intensa actividad excavadora, posiblemente rápida, con fines alimenticios (comportamiento de tipo fodinicnia) por parte de un crustáceo decápodo sedimentívoro, ya que el rastrillaje más o menos ordenado del sedimento así parece indicarlo. De manera secundaria, el organismo tendería a estabilizar la estructura con un fino revestimiento, que le permitiría usarla también como morada temporal. Estas características son congruentes con las madrigueras realizadas por los crustáceos decápodos, tal y como se ha observado en especies de callianásidos actuales (Flor et al., 1979; Ekdale et al., 1984; Bromley, 1990; Ekdale, 1992; entre muchos otros).

En el caso de Spongeliomorpha chevronensis nov. ichnosp., es posible incluso inferir el movimiento de excavación y avance del organismo a partir del modelo espigado de las marcas de rastrillaje que realiza (ver Fig. 4). Inicialmente, el organismo "arañaría” en sentido descendente desde la parte superior hasta aproximadamente la mitad de los flancos y después desde este punto, y en el mismo sentido, hacia la parte inferior. Este procedimiento dejaría un patrón en forma de espiga con las "uves" más pronunciadas en los flancos, que indicarían el sentido de avance (ver Fig. 4). Por otra parte, en Spongeliomorpha sinuostriata nov. ichnosp., el desplazamiento sólo se puede conocer cuando aparece en conexión con otra pista que tenga un referente claro de movimiento (Teichichnus, Fig. 7e). En este caso, el organismo excavaría la madriguera dejando marcas longitudinales más o menos continuas (ver Tabla 1) y de forma sucesiva, tal y como señalan sus cruces.

En ambos modelos de excavación, el sedimento extraído sería posiblemente transportado a los pereiópodos torácicos para digerir los nutrientes y desechar el resto, que transportaría hacia la parte posterior o aplicaría a la pared como revestimiento para afianzar la construcción de la galería con carácter semipermanente (Bromley, 1990; Ekdale, 1992; Muñiz et al., 1995; Muñiz Guinea, 1998).

Por otra parte, D’Alessandro y Bromley (1995) interpretan Spongeliomorpha sicula como una pista fósil de tipo agricnia donde el cultivo microbiano se realizaría en las cámaras ovoidales. Otras interpretaciones dadas por los autores anteriores son que las cámaras pudieron servir como áreas de reproducción y crianza de larvas (este comportamiento se ha observado muy bien en callianásidos actuales, como Callianassa kraussi Forbes, 1973. También podrían servir como áreas de acumulación de desechos cuando el sedimento hubiera entrado accidentalmente en las madrigueras. Una última interpretación la basan en el trazado geométrico del sistema, que podría servir para facilitar la captura de posibles presas, según el paradigma que rige este tipo de diseños (Seilacher, 1977). D'Alessandro y Bromley (1995) rechazan la posibilidad de que $S$. sicula pueda interpretarse como una pista de búsqueda de alimento (fodinicnion) realizada por organismos sedimentívoros; ya que sus ejemplares se encuentran en sedimentos muy consolidados, originalmente muy pobres en agua y materia orgánica (son cenizas volcánicas). Además, son sistemas muy profundos conectados entre sí y con la superficie mediante fustes que llegan a alcanzar hasta 40 $\mathrm{cm}$ de longitud, a través de los cuales realizarían un tipo de alimentación suspensívora.

En nuestro caso, todos los sistemas de S. sicula se encuentran en relación con fondos limo-fangosos, semiconsolidados, ricos en materia orgánica y que estarían ocupando los niveles inmediatamente por debajo de la interfase sedimento-agua. En estos limos, además, el grado de bioturbación es muy intenso (Cachão, 1995; Muñiz Guinea, 1998). La presencia de la ornamentación en el exterior de las paredes sugiere, como ya se indicó, una intensa actividad excavadora, con un rastrillaje más o menos ordenado del sedimento. Los nutrientes serían de esta forma acumulados en las cámaras para favorecer el crecimiento microbiano (agricnion), las cámaras ocasionalmente podrían ser usadas como áreas de nidificación. Ambos casos implicarían el uso del sistema de galerías como una morada semipermanente. La posibilidad de constituir un sistema de almacenaje de residuos parece bastante improbable, ya que al ser madrigueras poco profundas, podrían ser transportados fácilmente al exterior. Por último, la ausencia de una arquitectura geométrica más 
compleja impide hacer apreciaciones (al menos hasta el momento) sobre si pudieron ser construidos con la finalidad de capturar pequeñas presas.

Normalmente, la sección de Spongeliomorpha suele ser circular a elipsoidal; sin embargo, en el caso particular de S. sinuostriata nov. ichnosp., se ha podido observar que varía de hexagonal-subhexagonal a subelipsoidal. Este hecho anómalo tiene su explicación en fenómenos de compactación diferencial (Fig. 6), ya que todas las secciones anteriores pueden aparecer a lo largo de una misma madriguera. Las secciones poligonales (Fig. 6C) corresponderían a las zonas más deformadas.

\section{CONTEXTO PALEOAMBIENTAL}

La presencia del icnogénero Spongeliomorpha sugiere diversas interpretaciones relativas al (paleo)ambiente en el que fueron realizadas. Se suelen encontrar tanto en medios marinos (Calzada, 1981) como en medios acuáticos no marinos (Bromley y Aasgard, 1979; Pickerill, 1992; Metz, 1993, 1995). En particular, las icnoespecies estudiadas se encuentran en la parte alta del conjunto de limos arenosos blancos de la serie miocena (ver Fig. 2). Según Muñiz Guinea (1998), Muñiz et al. (1998a) y Mayoral y Muñiz (2000), este conjunto litológico representa un medio confinado (bahía), de baja energía, rico en materia orgánica, de salinidad marina normal y con fondos limo-fangosos consolidados a muy consolidados, como refleja la conservación de todo tipo de ornamentación (marcas de rastrillaje) en las pistas, sobre todo en Spongeliomorpha; dato que está ampliamente referido en la bibliografía (Calzada, 1981; Kennedy, 1967; Bromley y Asgaard, 1979; Ekdale et al., 1984; Bromley, 1990; Metz, 1993, 1995; D’Alessandro y Bromley, 1995; entre muchos otros). Las condiciones batimétricas en la parte inferior de la serie corresponden a la franja litoral en la que se han interpretado (Muñiz Guinea, 1998) frecuentes reactivaciones de carácter transgresivo, mientras que en la parte media-alta, correspondiente a las franjas infralitoral superior y litoral, las reactivaciones son regresivas. Desde el punto de vista paleoicnológico, en el tramo limoso (Tramo 2) donde aparece Spongeliomorpha se han reconocido treinta y nueve icnoespecies pertenecientes a veinte icnogéneros (Arenicolites, Bichordites, Cardioichnus, Conichnus, Cylindrichnus, Dactyloidites, Gyrolithes, Lithoplaision, Macanopsis, Ophiomorpha, Palaeophycus, Phycodes, Psilonichnus, Rosselia, Scalarituba, Scolicia, Skolithos, Taenidium, Teichichnus, Thalassinoides); así como varias estructuras de cubicnia y otras relacionadas con posibles Planolites. El nivel medio-superior de este tramo está caracterizado por la icnoasociación de SpongeliomorphaTeichichnus-Phycodes (según Muñiz Guinea, 1998 y Muñiz et al, 1998a), que señala un dominio de las estructuras etológicas de tipo fodinicnia sobre las de domicnia, pascicnia y cubicnia, estando relacionadas con organismos sedimentívoros y suspensívoros (crustáceos decápodos, anélidos, equinodermos y anémonas, principalmente).
Por otra parte, Spongeliomorpha sinuostriata nov. ichnosp. también aparece, aunque con menor abundancia (ver Fig. 2), en el nivel superior del conjunto de limosarenosos del Tramo 8 de la serie pliocena (Muñiz y Mayoral, 1998). Estas litofacies representan, en conjunto, un medio confinado (estuario) de carácter marino más abierto hacia techo. La parte inferior del tramo correspondería a la zona más profunda del estuario y las restantes, a su colmatación progresiva dominadas por un régimen intermareal, de salinidad salobre y energía cada vez mayor (Muñiz Guinea, 1998; Muñiz et al., 1998a, Mayoral y Muñiz, 1998). En este tramo, se han reconocido, junto a Spongeliomorpha sinuostriata, dieciocho icnoespecies pertenecientes a once icnogéneros: Arenicolites, Conichnus, Cylindrichnus, Gyrolithes, Palaeophycus, Psilonichnus, Scalarituba, Skolithos, Taenidium, Teichichnus y Thalassinoides; así como varias estructuras de cubicnia. El nivel donde se localiza $S$. sinuostriata está caracterizado por la icnoasociación de Gyrolithes variabilis (Muñiz Guinea, 1998; Muñiz et al., 1998a, Muñiz y Mayoral, 1998) con predominio de las estructuras etológicas de domicnia sobre las de fodinicnia, pascicnia y cubicnia, realizadas mayoritariamente por organismos sedimentívoros (crustáceos decápodos y anélidos).

\section{CONCLUSIONES}

El estudio de las pistas fósiles en los depósitos marinos neógenos del sector suroccidental de la Cuenca del Guadalquivir (Lepe-Ayamonte, Huelva, España) ha permitido la identificación de varias icnoespecies relacionadas con el icnogénero Spongeliomorpha: $S$. sicula y $S$. ichnosp. indet., encontradas en los limos arenosos de edad Mioceno (Tortoniense) (Tramo 2 de la serie litoestratigráfica global), y dos nuevas icnoespecies, denominadas $S$. chevronensis y $S$. sinuostriata que aparecen en los limos miocenos, encontrándose la última de ellas también en la serie pliocena (Tramo 8). Las características morfológicas que definen las nuevas icnoespecies de Spongeliomorpha se refieren principalmente al patrón ornamental que presentan las marcas de rastrillaje conservadas en las paredes exteriores. En concreto, Spongeliomorpha chevronensis presenta las marcas dispuestas en forma de espiga (chevron) y dispuestas oblicuamente respecto al eje principal de la madriguera. Spongeliomorpha sinuostriata presenta las marcas yuxtapuestas, su trayectoria es sinuosa y forman un enrejado sigmoidal, siendo longitudinales respecto al eje principal. Estos patrones ornamentales las diferencian claramente de las otras icnoespecies encontradas hasta la fecha, lo que apoya su propuesta como icnoespecies nuevas.

\section{AGRADECIMIENTOS}

Al Dr. A. Uchman (Institute of Geological Sciences Jagiellonian University, Cracovia, Polonia) y a D. J.A. Gámez 
Vintaned (Universidad de Badajoz) por sus comentarios críticos al manuscrito. Al Dr. J.J. Álvaro (Université de Sciences et Technologies de Lille, Francia) por la información suministrada. A los Drs. Jordi Martinell Callicó (Universidad de Barcelona), Eladio Liñán Guijarro (Universidad de Zaragoza), Sixto Fernández-López (Universidad Complutense de Madrid) y María Luisa Martínez Chacón (Universidad de Oviedo) por la revisión crítica del manuscrito y las sugerencias recibidas. Este trabajo se ha realizado dentro del marco del Grupo de Investigación n. ${ }^{\circ}$ RNM 0219 "G.I. NATURALAND" de la Junta de Andalucía y del Proyecto de la D.G.I.C.Y.T. PB-94-0946.

\section{BIBLIOGRAFÍA}

Azpeitia Moros, F. 1933. Datos para el estudio paleontológico: el Flysch de la Costa Cantábrica y de algunos otros puntos de España. Boletín del Instituto Geológico y Minero de España, 53, 1-65.

Bosca, E. 1917. A propósito del Taonurus ultimus vel Spongeliomorpha iberica Saporta. Boletin de la Sociedad Española de Historia Natural, 17, 263-268.

Bromley, R.G. 1990. Trace fossils: Biology and Taphonomy. Unwin Hyman, London, 280 pp.

Bromley, R.G. and Aasgard U. 1979. Triassic freshwater ichnocoenoses from Carlsberg Fjord, East Greenland. Palaeogeography, Palaeoclimatology, Palaeoecology, 28, 39-80.

Bromley, R.G. and Frey, R.W. 1974. Redescription of the trace fossils Gyrolithes and taxonomic evaluation of Thalassinoides, Ophiomorpha and Spongeliomorpha. Geological Society of Denmark Bulletin, 23, 311-335.

Cáceres Puro, L.M. 1999. Geomorfología del sector occidental de la Depresión del Guadalquivir (Huelva). Servicio de Publicaciones de la Universidad de Huelva, 250 pp.

Cachão, M. 1995. Utilização de Nanofósseis calcários em Biostratigrafia, Paleoceanografía e Paleoecologia. Aplicações ao Neogenico do Algarve (Portugal) e do Mediterrãneo Ocidental (ODP 653) e à problemática de Coccolithus pelagicus. Tesis Doctoral (inédita). Universidad de Lisboa, 356 pp.

Calzada, S. 1981. Revisión del icno Spongeliomorpha iberica Saporta, 1887 (Mioceno de Alcoy, España). Boletín de la Real Sociedad Española de Historia Natural (Geologica), 79. 189-195.

Chiplonkar, G.W. and Ghare, M.A. 1975, Some additional trace fossils from the bagh beds. Bulletin of Indian Geological Association, 8(1), 71-84.

D'Alessandro, A. and Bromley, R.G. 1995. A new ichnopecies of Spongeliomorpha from the Pleistocene of Sicily. Journal of Paleontology, 69, 393-398.

Darder, B. 1945. Estudio geológico de la provincia de Valencia y norte de la de Alicante. Boletín del Instituto Geológico y Minero de España, 57, 59-362.

Ekdale, A.A. 1992. Muckraking and Mudslinding: the Joys of Deposit-Feeding. In: Trace fossils (Eds. Ch. G. Maples and R.R. West) Short Courses in Paleontology, 5, 145-171.
Ekdale, A.A., Bromley, R.G. and Pemberton, G.S. 1984. Ichnology: the use of trace fossils in sedimentology and stratigraphy. Society of Economic Paleontologists and Mineralogists, Short Course in Paleontology, 15, 1-317.

Eyles, CH. H., Eyles, N. and Gostin, V.A. 1998. Facies and allostratigraphy of high-latitude, glacially influence marine strata of the early Permian Southern Sydney Basin, Australia. Sedimentology, 45, 121-161.

Flor, G, García-Ramos, J.C. y Valenzuela, M. 1979. Actividad biogénica en los depósitos actuales de la playa de San Pedro de Antromero (Asturias). Boletín de la Real Sociedad Española de Historia Natural (Geología), 77, 181-197.

Forbes, A.T. 1973. An unusual abbreviated larval life in the estuarine burrowing prawn Callianassa kraussi (Crustacea: Decapoda: Thalassinidea). Marine Biology, 22, 361-365.

Fuchs, T. 1895. Studien über Fucoiden und Hieroglyphen. Denkschr Akademie der Wissenschaften zu Wien, Mathematisch-Naturwissenschaftliche klasse, Denkschriften, 62, 369-448.

Fürsich, F.T. 1973. A revision of the trace fossils Spongeliomorpha, Ophiomorpha and Thalassinoides. Neues Jahrbuch für Geologie und Paläontologie, Abhandlungen; Beilage-Bände; Monatshefte, 12, 719735.

Galán, E., González, I., Mayoral, E. and Muñiz, F. 1995. Contribution of clay mineralogy to the paleoenvironmental interpretation of upper Miocene detrital sediments. Abstracts Euroclay'95. Leuven, Bélgica, 311-312.

Gale, A.S. 1996. Turonian correlation and sequence stratigraphy of the Chalk in southern England. In: Sequence Stratigraphy in British Geology (Eds. S.P. Hasselbo and D.N. Parkinson). Geological Society, London, 177-195.

Ghibaudo, G., Grandesso, P., Massari, F. and Uchman, A. 1996, Use of trace fossils in delineating sequence stratigraphic surfaces (Tertiary Venetian Basin, northeastern Italy). Palaeogeography, Palaeoclimatlogy, Palaeoecology, 120, 261-279.

Kennedy, W.J. 1967. Burrows and surface traces from the Lower Chalk of Southern England. Bulletin of The British Museum (Natural History), Geology, 15 (3), 125 167.

Kennedy, W.J. and McDougall, J. D. 1969. Crustacean burrows in the Weald Clay (Lower Cretaceous) of SouthEarsten England and their enviromental significance. Paleontology, 12, 459-471.

Książkiewicz, M. 1961. On some problematic organic traces from the flysch of the Polish Carpathians (part II). (In Polish, English summary). Kwartalnik Geologiczny, 5, 882-890.

Książkiewicz, M. 1977. Trace fossils in the Flysch of the Polish Carpathians. Paleontologica Polonica, 36, 1-208.

Laubenfels, M.W. de. 1955. Porifera. In: Treatise on Invertebrate Paleontology (Ed. R.C. Moore). Geological Society of America \& University of Kansas Press. New York \& Lawrence, Kansas, part E, 21-112. 
Lesertisseur, J. 1955. Traces fossiles d'activitié animale et leur significance paléobiologique. Société Géologique de la France, 74, 1-148.

Marcinowski, R. and Wierzowski, A. 1975. On the nature of decapod burrows Spongia sudolica of Zarìczny (1878). Acta Geologica Polonica, 25, 399-405.

Mayoral, E. y Muñiz, F. 1994. Presencia de un nuevo cefalópodo sepioideo en el Neógeno superior de la Cuenca del Guadalquivir (Lepe, Huelva, España). Coloquios de Paleontología, 46, 161-174.

Mayoral, E. y Muñiz, F. 1995. Nueva icnoespecie de Gyrolithes del Mioceno superior de la Cuenca del Guadalquivir (Lepe, Huelva). Revista Española de Paleontología, 10, 190-201.

Mayoral. E. y Muñiz, F. 1996. La Icnofacies de Gnathichnus en el sector Suroccidental de la Cuenca del Guadalquivir (Lepe, Huelva, España). Coloquios de Paleontología, 48, 71-82.

Mayoral, E. y Muñiz, F. 1998. Nuevos datos icnotaxonómicos sobre Gyrolithes del Plioceno inferiorsuperior de la Cuenca del Guadalquivir (Lepe, Huelva, España). Revista Española de Paleontología, 13, 61-69.

Mayoral, E. and Muñiz, F. 2000. New ichnospecies of Cardioichnus from the Miocene of the Guadalquivir Basin, Huelva, Spain. Ichnos.

Metz, R. 1993. A new ichnospecies of Spongeliomorpha from the Late Triassic of New Jersey. Ichnos, 2, 259-263.

Metz, R. 1995, Ichnologic study of the Lockatong Formation (Late Triassic), Newark Basin, southeastern Pennsylvania. Ichnos, 4, 43-52.

Muñiz Guinea, F. 1998. Paleoicnología del Neógeno Superior en el Sector Suroccidental de la Cuenca del Guadalquivir, Área de Lepe-Ayamonte (Huelva). Tesis Doctoral (inédita), Universidad de Huelva, $272 \mathrm{pp}$.

Muñiz, F. and Mayoral, E. 1998. Paleoichnology of the lower Pliocene estuarine facies in the SW sector of the Guadalquivir basin (Lepe-Ayamonte area, Huelva, Spain). $1^{\text {st }}$ Interdisciplinary Symposium on Estuarine Processes, Faro, Portugal, 72-76.

Muñiz, F., Mayoral, E. y Martín, M. 1995. Pistas fósiles intergradacionales en el Mioceno superior del sector suroccidental de la Cuence del Guadalquivir. Libro de Comunicaciones de las XI Jornadas de Paleontología, Tremp (Lérida), 119-122.

Muñiz, F., Mayoral, E. y Gámez Vintaned, J.A. 1998a. Icnofacies del Neógeno superior de la Cuenca del Guadalquivir (área de Lepe-Ayamonte, Huelva, españa). Geogaceta, 24, 235-238.

Muñiz, F., Mayoral, E. y Rodríguez, J. 1998b. Nuevo registro de Spongeliomorpha sicula (D'Alessandro y Bromley, 1995) en el Mioceno superior de la Península Ibérica. Libro de Comunicaciones de las XIV Jornadas de Paleontología, Tenerife, 135-138.

Muñiz, F., Mayoral, E., Barrón, E. y Cachão, M. 1999.
Nuevos datos sobre macroflora del Plioceno en el suroeste de la Península Ibérica (Lepe, Huelva, España). Geogaceta, 25, 143-146.

Mutti, M., Bernouilli, D., Eberli, G.P. and Vecsei, A. 1996. Depositional geometries and facies associations in an Upper Cretaceous prograding carbonate plataform margin (Orfento Supersequence, Maiella, Italy). Journal of Sedimentary Research, 66, 749-765.

Okada, H. and Bukry, D. 1980. Supplementary modification and introduction of code numbers to the low-latitude coccolith biostratigraphic zonation (Bukry, 1973, 1979). Marine Micropaleontology, 5(3), 171-187.

Pickerill, R. 1992. Carboniferous nonmarine invertebrate ichnocoenoses from southern New Brunswick, Eastern Canada. Ichnos, 2, 21-35.

Saporta, G. de 1887. Nouveaux documents relatifs aux organismes problématiques des anciennes mers. Masson, Paris, 100 pp.

Schimper, W. Ph. 1869. Traité de Paléontologie végetale ou la flore du monde primitif. 1, 740 pp., 2: 522 pp., 3: 896 pp.

Seilacher, A. 1977. Evolution of Trace Fossil Communities. In: Patterns of evolution, as illustrated by the fossil record, 11 (Ed. A. Hallam), 359-376.

Sierro, F.J., González-Delgado, J.A., Flores, J.A., Dabrio, C.J., and Civis, J. 1990. The Neogene of the Guadalquivir Basin (SW Spain). Paleontologia $i$ Evolució, 2, 209-250.

Uchman, A. 1998. Taxonomy and ethology of flysch trace fossils: revision of the Marian Ksi $\varnothing$ kiewicz collection and studies of complementary material. Annales Societatis Geologorum Poloniae, 68, 105-218.

Uchman, A. and Álvaro. J.J. 1999. A case study of ichnofabrics from the Miocene lacustrine deposits of the Calatayud-Teruel Basin, NE Spain. Abstract of $5^{\text {th }}$ International Ichnofabric Workshop IIW5, University of Mancherster, 12-14.

Uchman, A. and Álvaro, J.J. 2000. Non-marine invertebrate trace fossils from the Tertiary Calatayud-Teruel basin, NE Spain. Revista Española de Paleontología, 15, 203-218.

Viguier, C. 1974. Le Neogené en Andalousie NordOccidentale (Espagne). Histoire géologique de "Bassin du Bas-Guadalquivir”. Thèse Doctoral (inédita), Université de Bordeaux, 450 pp.

Weigelt, J. 1929. Fossile Grabschächte brachyurer Decapoden als Lokalgeschriebe in Pommern und das Rhizocorallium problem. Zeitschrift für Geschiebeforsch, 5(1-2), 1-42.

Zonneveld, J.P., Saunders, T.D.A., Pemberton, S.G., Gingras, M.K. and Brett, K.D. 1998. Sediment-organism zonation and the recolonization of Triassic shorelines, Williston Lake, Northeastern British Columbia. Abstract of $15^{\text {th }}$ International Sedimentological Congress, Alicante, 846-847. 